\title{
On Energy Conservation in Data Centers
}

\author{
SUSANNE ALBERS, Technical University of Munich
}

\begin{abstract}
We formulate and study an optimization problem that arises in the energy management of data centers and, more generally, multiprocessor environments. Data centers host a large number of heterogeneous servers. Each server has an active state and several standby/sleep states with individual power consumption rates. The demand for computing capacity varies over time. Idle servers may be transitioned to low-power modes so as to rightsize the pool of active servers. The goal is to find a state transition schedule for the servers that minimizes the total energy consumed. On a small scale, the same problem arises in multicore architectures with heterogeneous processors on a chip. One has to determine active and idle periods for the cores so as to guarantee a certain service and minimize the consumed energy.

For this power/capacity management problem, we develop two main results. We use the terminology of the data center setting. First, we investigate the scenario that each server has two states: an active state and a sleep state. We show that an optimal solution, minimizing energy consumption, can be computed in polynomial time by a combinatorial algorithm. The algorithm resorts to a single-commodity minimum-cost flow computation. Second, we study the general scenario that each server has an active state and multiple standby/sleep states. We devise a $\tau$-approximation algorithm that relies on a two-commodity minimum-cost flow computation. Here, $\tau$ is the number of different server types. A data center has a large collection of machines but only a relatively small number of different server architectures. Moreover, in the optimization, one can assign servers with comparable energy consumption to the same class. Technically, both of our algorithms involve nontrivial flow modification procedures. In particular, given a fractional two-commodity flow, our algorithm executes advanced rounding and flow packing routines.
\end{abstract}

CCS Concepts: • Theory of computation $\rightarrow$ Design and analysis of algorithms; Approximation algorithms analysis; Scheduling algorithms; Discrete optimization; • Hardware $\rightarrow$ Power estimation and optimization; Enterprise level and data centers power issues;

Additional Key Words and Phrases: Heterogeneous machines, efficient algorithms, approximation algorithms, minimum-cost flow

\section{ACM Reference format:}

Susanne Albers. 2019. On Energy Conservation in Data Centers. ACM Trans. Parallel Comput. 6, 3, Article 13 (October 2019), 26 pages.

https://doi.org/10.1145/3364210

\section{INTRODUCTION}

We define and investigate an optimization problem with the objective of energy conservation in multiprocessor environments. We focus on two particularly timely settings.

This work was supported by the European Research Council, Grant Agreement No. 691672.

Authors' address: S. Albers, Technical University of Munich, Boltzmannstr. 3, Garching, 85748, Germany; email: albers@ in.tum.de.

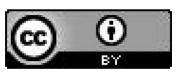

This work is licensed under a Creative Commons Attribution International 4.0 License.

(C) 2019 Copyright held by the owner/author(s).

2329-4949/2019/10-ART13

https://doi.org/10.1145/3364210 
Data centers. Energy management is a key issue in data center operations [8]. Electricity costs are a dominant and rapidly growing expense in such centers; about $30 \%$ to $50 \%$ of their budget is invested into energy. Data centers use about $1.5 \%$ of the total electricity worldwide [14]. This corresponds to the energy consumption of more than 90 million households [10]. Surprisingly, the servers of a data center are only utilized $20 \%$ to $40 \%$ of the time on average [2,5]. When idle and in active mode, they consume about half of their peak power. Hence, a fruitful approach for energy conservation and capacity management is to transition idle servers into standby and sleep states. Servers have a number of low-power states [7]. However, state transitions, particularly powerup operations, incur energy consumption (cost). Therefore, dynamically matching the varying demand for computing capacity with the number of active servers is a challenging problem.

Multicore architectures. Multicore processors are architectures with multiple and often heterogeneous processing units on a single die. Originally, heterogeneous platforms contained several processor types, i.e., CPUs and GPUs. Modern platforms are also equipped with identical CPUs that have different microarchitectures leading to various levels of energy consumption [6]. To exploit such platforms, effective power management strategies are needed. The optimization problem is identical to that described in the last paragraph, except we have a small number of processing units here. We remark that in such environments, the processors might have several active states as well.

In Section 2, we formally define an optimization problem, Dynamic Power Management (DPM), that captures the preceding scenarios. In short, there are $m$ heterogeneous servers (processors). Each server has several states with associated power consumption rates. State transitions consume energy. The planning horizon contains times $t_{1}<t_{2}<\cdots<t_{n}$ at which the demand changes. During interval $\left[t_{k}, t_{k+1}\right)$, at least $d_{k}$ servers must be active and available for utilization, $1 \leq k \leq$ $n-1$. The goal is to find a state transition schedule for the servers minimizing the total energy consumption.

\subsection{Previous Work}

Augustine et al. [3] and Irani et al. [15] study power-down strategies for a single device that is equipped with an active state and several low-power states. The goal is to minimize the energy consumed in an idle period. Our problem DPM is a generalization with multiple, parallel devices and time-dependent demand. The two articles $[3,15]$ develop online algorithms that achieve optimal competitive ratios. DPM for a single device with two states is equivalent to the ski-rental problem, a famous rent-or-buy problem [16, 17, 19, 22]. No generalization with several required resources has been examined. Azar et al. [4] study a capital investment problem where machines for manufacturing a product may be purchased over time. The machines differ in the capital and production costs.

Khuller et al. [18] and Li and Khuller [20] introduce machine activation problems that are also motivated by energy conservation in data centers. In Khuller et al. [18], the authors assume that there is an activation cost budget, and jobs have to be scheduled on the selected, activated machines so as to minimize the makespan. They present algorithms that simultaneously approximate the budget and the makespan. The second work [20] considers a generalization where the activation cost of a machine is a nondecreasing function of the load. Demaine et al. [9] investigate the problem of scheduling unit-size jobs on a set of identical parallel processors having one sleep state. Each job can be executed in any of a set of given time intervals. For the objective of minimizing power consumption, they develop an approximation algorithm whose performance factor depends on the energy needed to transition between the active state and the sleep state.

In the more applied computer science literature, power management strategies and the value of sleep states have been studied extensively. The works mostly focus on experimental evaluations. 
Analytic results are presented in other articles as well [11-13, 21, 23]. Ghandi et al. [12] model a server farm with setup costs as an $M / M / m$ queuing system. Lin et al. [21] study a dynamic rightsizing of data centers with homogeneous servers having one sleep state. The operating cost of a server is a convex function of the workload.

\subsection{Our Contribution}

We present an algorithmic study of an important capacity management problem in data centers. Our problem DPM dynamically rightsizes the pool of servers with the objective to minimize the energy consumed. Compared to previous work, the new, essential aspects are that we consider (a) a time horizon with varying demand for computing capacity and (b) power-heterogeneous servers. In fact, with homogeneous servers, the problem is easy to solve. In DPM, the demand for computing capacity is specified by the number of servers needed at any time. In data centers, it is common practice that a number of required servers is determined as a function of the current total workload, ignoring specific jobs. DPM focuses on energy conservation instead of individual job placement.

We investigate DPM as an offline problem, i.e., the varying computing demands are known in advance. From an algorithmic point of view, it is important to explore the tractability and approximability of the problem. The offline setting is also relevant in practice. Data centers usually analyze past workload traces to identify long-term patterns. The findings are used to specify demands in future time windows.

In Section 3, we study DPM in the scenario that each server has two states: one active state and one sleep state. This is a basic setting that, in a first step, abstracts away the full spectrum of low-power modes. Most of the more applied literature on power management strategies assumes the existence of a single sleep state. We show that DPM can be solved in polynomial time by a combinatorial algorithm. We devise an algorithm that resorts to a single-commodity minimumcost flow computation. In the corresponding network, there is a component for each server. Such a component contains an upper path and a lower path, representing the server's active state and sleep state, respectively. Unfortunately, an arbitrary minimum-cost flow does not correspond to a feasible schedule. Our algorithm modifies flow so that an optimal schedule can be derived.

In Section 4, we investigate DPM in the general scenario that each server has multiple sleep states. We extend our approach based on flow computations. We develop a second algorithm that works with a more complex network in which each component has several lower paths, representing the various low-power states of a server. Furthermore, we need a second commodity to ensure that computing demands are met. With only a single commodity, flow units could switch between lower paths at no cost, and infeasible schedules would result. Given a fractional two-commodity minimum-cost flow, our algorithm executes advanced flow rounding and packing procedures. First, by repeatedly traversing components, the algorithm modifies flow so it becomes integral on the upper paths. Then flow on the lower paths is packed. The final integral flow allows the constructing of a schedule for DPM. Our algorithm achieves an approximation factor of $\tau$, where $\tau$ is the number of server types in the problem instance. The servers can be partitioned into $\tau$ classes such that, within each class, the servers are identical. Of course, the servers of a class are independent and not synchronized. In practice, a data center has a large collection of machines but a relatively small number of different server architectures. Furthermore, in the optimization, machines with comparable energy consumption characteristics can be assigned to the same server class.

We note that our algorithms can handle the problem extension that the power consumption rates are time dependent. This can model, e.g., scenarios in which servers are temporarily unavailable due to maintenance or because they are reserved for other tasks. 


\section{PRELIMINARIES}

\subsection{The Problem DPM}

A problem instance $\mathcal{I}=(\mathcal{S}, \mathcal{D})$ is specified by a set of servers and varying computing demands over a time horizon. Let $\mathcal{S}=\left\{S_{1}, \ldots, S_{m}\right\}$ be a set of heterogeneous servers. Each server $S_{i}, 1 \leq i \leq$ $m$, has an active state, as well as one or several standby/sleep states. The states of $S_{i}$ are denoted by $s_{i, 0}, \ldots, s_{i, \sigma_{i}}$. Here, $s_{i, 0}$ is the active state and $s_{i, 1}, \ldots, s_{i, \sigma_{i}}$ are the low-power states. The modes have individual power consumption rates. Let $r_{i, j}$ be the power consumption rate of $s_{i, j}$, i.e., $r_{i, j}$ energy units are consumed per time unit while $S_{i}$ resides in $s_{i, j}$. The states are numbered in order of decreasing rates such that $r_{i, 0}>\cdots>r_{i, \sigma_{i}} \geq 0$. A server can transition between its states. Let $\Delta_{i, j, j^{\prime}}$ be the non-negative energy needed to move $S_{i}$ from state $s_{i, j}$ to state $s_{i, j^{\prime}}$, for any pair $1 \leq$ $j, j^{\prime} \leq \sigma_{i}$. The transition energies satisfy the triangle inequality, i.e., the energy to move directly from $s_{i, j}$ to $s_{i, j^{\prime}}$ is upper bounded by that of visiting an intermediate state $s_{i, k}$. Formally, $\Delta_{i, j, j^{\prime}} \leq$ $\Delta_{i, j, k}+\Delta_{i, k, j^{\prime}}$, for any $j, j^{\prime}, k$.

Over a time horizon, the computing demands are given by a demand profile $\mathcal{D}=(T, D)$. Tuple $T=\left(t_{1}, \ldots, t_{n}\right)$ contains the points in time when the computing demands change. There holds $t_{1}<$ $t_{2}<\cdots<t_{n}$ so that the time horizon is $\left[t_{1}, t_{n}\right)$. Tuple $D=\left(d_{1}, \ldots, d_{n-1}\right)$ specifies the demands. More precisely, $d_{k} \in \mathbb{N}_{0}$ servers are required for computing during interval $\left[t_{k}, t_{k+1}\right)$, for any $1 \leq$ $k \leq n-1$. Thus, at least $d_{k}$ servers must reside in the active state during $\left[t_{k}, t_{k+1}\right)$. We have $d_{k} \leq m$, for any $1 \leq k \leq n-1$, so that the requirements can be met.

Given $\mathcal{I}=(\mathcal{S}, \mathcal{D})$, a schedule $\Sigma$ specifies, for each $S_{i}$ and any $t \in\left[t_{1}, t_{n}\right)$, in which state server $S_{i}$ resides at time $t$. Schedule $\Sigma$ is feasible if during any interval $\left[t_{k}, t_{k+1}\right)$ at least $d_{k}$ servers are in the active state, $1 \leq k \leq n-1$. The energy $E(\Sigma)$ incurred by $\sum$ is the total energy consumed by all $m$ servers. Whenever server $S_{i}, 1 \leq i \leq m$, resides in state $s_{i, j}$, it consumes energy at a rate of $r_{i, j}$. Whenever the server transitions from state $s_{i, j}$ to state $s_{i, j^{\prime}}$, the incurred energy is $\Delta_{i, j, j^{\prime}}$. The goal is to find an optimal schedule, i.e., a feasible schedule $\Sigma$ that minimizes $E(\Sigma)$. We assume that initially, immediately before $t_{1}$, and at time $t_{n}$, all servers reside in the deepest sleep state, i.e., $S_{i}$ is in $s_{i, \sigma_{i}}, 1 \leq i \leq m$. Our algorithms and results can be adapted easily to the setting where each server initially/finally takes arbitrary desired states.

\subsection{Properties of Optimal Schedules}

Given a problem instance $\mathcal{I}$, we characterize optimal schedules. Proposition 2.1 implies that there exists an optimal schedule in which a server never changes state while being in low-power mode. Of course, the low-power states may vary for the various intervals in which a server is not active. Proposition 2.2 states that there exists an optimal schedule executing state transitions only when the computing demands change. A server powers up if it transitions from a low-power state to the active state. A server powers down if it moves from the active state to a low-power state. The proofs of Propositions 2.1 and 2.2 are given in the appendix.

Proposition 2.1. There exists an optimal schedule with the following property. Suppose that $S_{i}$ powers down at time $t$ and next powers up at time $t^{\prime}$. Then between $t$ and $t^{\prime}, S_{i}$ resides in a single state $s_{i, j}$, where $j>0$. At time $t, S_{i}$ transitions directly from $s_{i, 0}$ to $s_{i, j}$. At time $t^{\prime}$, it moves directly from $s_{i, j}$ to $s_{i, 0}$.

Proposition 2.2. There exists an optimal schedule that satisfies the property of Proposition 2.1 and performs state transitions only at the times of $T$.

We finally argue that without loss of generality, the power-down energies $\Delta_{i, 0, j}$ are equal to 0 , $1 \leq i \leq m$ and $1 \leq j \leq \sigma_{i}$. We will always focus on optimal schedules with the property given in Proposition 2.1. At times $t_{1}$ and $t_{n}$, every server is in its deepest sleep state. The first time server 
$S_{i}$ moves to the active state, the least energy is consumed if it transitions directly from $s_{i, \sigma_{i}}$ to $s_{i, 0}$. The last time $S_{i}$ powers down, the best option is to move directly from $s_{i, 0}$ to $s_{i, \sigma_{i}}$. Hence, every server $S_{i}$ performs the same number of transitions from $s_{i, 0}$ to $s_{i, j}$ as from $s_{i, j}$ to $s_{i, 0}$, for any $1 \leq j \leq \sigma_{i}$. For any server $S_{i}$, only energies $\Delta_{i, 0, j}$ and $\Delta_{i, j, 0}, 1 \leq j \leq \sigma_{i}$, are relevant. Therefore, if $\Delta_{i, 0, j}>0$, we can add this energy to $\Delta_{i, j, 0}$, i.e., $\Delta_{i, j, 0}^{\prime}:=\Delta_{i, 0, j}+\Delta_{i, j, 0}$ and $\Delta_{i, 0, j}^{\prime}:=0$.

\section{SERVERS WITH TWO STATES}

We consider the variant of DPM in which each server $S_{i}$ has exactly two states: an active state $s_{i, 0}$ and a sleep state $s_{i, 1}, 1 \leq i \leq m$.

TheOREM 3.1. Let $I$ be an instance of DPM in which each server has exactly two states. An optimal schedule for I can be computed in polynomial time by a combinatorial algorithm that uses a minimum-cost flow computation.

In the remainder of this section, we prove Theorem 3.1. We first argue that we may assume without loss of generality that the power consumption rates in the sleep states are equal to 0 . More specifically, for any problem instance $\mathcal{I}$, an optimal schedule can be derived from an optimal solution to a modified instance $I^{\prime}$ in which the power consumption rates in the sleep states are indeed 0 . Formally, given $\mathcal{I}=(\mathcal{S}, \mathcal{D})$, define an instance $\mathcal{I}^{\prime}=\left(\mathcal{S}^{\prime}, \mathcal{D}\right)$. Set $\mathcal{S}^{\prime}$ consists of servers $S_{1}^{\prime}, \ldots, S_{m}^{\prime}$, where each server $S_{i}^{\prime}$ has again an active state and a sleep state. For any $S_{i}^{\prime}$, let $r_{i, 0}^{\prime}=r_{i, 0}-r_{i, 1}$ and $r_{i, 1}^{\prime}=0$, i.e., the rates are reduced by $r_{i, 1}$. All other problem parameters of $I^{\prime}$, namely the state transition energies and the demand profile, are identical to those of $\mathcal{I}$. The next proposition states that an optimal schedule for $\mathcal{I}$ translates into an optimal schedule for $\mathcal{I}^{\prime}$ and vice versa. Only the consumed energy differs by $\sum_{i=1}^{m} r_{i, 1}\left(t_{n}-t_{1}\right)$.

Proposition 3.2. Any schedule $\Sigma$ for $I$ that is executed for $I^{\prime}$ consumes an energy of $E(\Sigma)-$ $\sum_{i=1}^{m} r_{i, 1}\left(t_{n}-t_{1}\right)$. Any schedule $\Sigma^{\prime}$ for $\mathcal{I}^{\prime}$ that is executed for $\mathcal{I}$ consumes an energy of $E\left(\Sigma^{\prime}\right)+$ $\sum_{i=1}^{m} r_{i, 1}\left(t_{n}-t_{1}\right)$

Proof. Considering that the demand profiles of $\mathcal{I}$ and $\mathcal{I}^{\prime}$ are identical, any schedule for $\mathcal{I}$ can be executed for $I^{\prime}$ and vice versa. In $\mathcal{I}^{\prime}$, for any server $S_{i}^{\prime}$ with $1 \leq i \leq m$, the power consumption rates in the active and sleep states are reduced by $r_{i, 1}$, compared to those of $S_{i}$. In addition, considering that at any time a server either resides in the active state or the sleep state, the proposition follows.

In the following, let $\mathcal{I}=(\mathcal{S}, \mathcal{D})$ be a problem instance in which the power consumption rates in the servers' sleep states are 0 . To simplify notation, let $r_{i}:=r_{i, 0}$ be the power consumption rate of $S_{i}$ in the active state, $1 \leq i \leq m$. Moreover, let $\Delta_{i}:=\Delta_{i, 1,0}$ be the energy needed to transition $S_{i}$ from the sleep state to the active state. We develop an algorithm $\mathcal{A}_{1}$ that computes an optimal schedule. Based on Proposition 2.2, we focus on schedules that perform state transitions only at the times of $T$. Given $\mathcal{I}=(\mathcal{S}, \mathcal{D}), \mathcal{A}_{1}$ constructs a network $\mathcal{N}(\mathcal{I})$. Any feasible schedule $\Sigma$ for $\mathcal{I}$ translates into a feasible flow of cost $E(\Sigma)$ in $\mathcal{N}(\mathcal{I})$. Any feasible flow of cost $C$ in $\mathcal{N}(\mathcal{I})$ can be converted so that it corresponds to a feasible schedule consuming energy $C$. The conversion requires some work but can be performed in a polynomial number of steps.

\subsection{Construction of the Network}

Consider any input $\mathcal{I}=(\mathcal{S}, \mathcal{D})$.

Network components. Network $\mathcal{N}(\mathcal{I})$ contains a component $C_{i}$, for each server $S_{i}, 1 \leq i \leq m$. Such a component $C_{i}$, which is depicted in Figure 1, consists of an upper path and a lower path. The upper path represents the active state of $S_{i}$; the lower path models the server's sleep state. The 


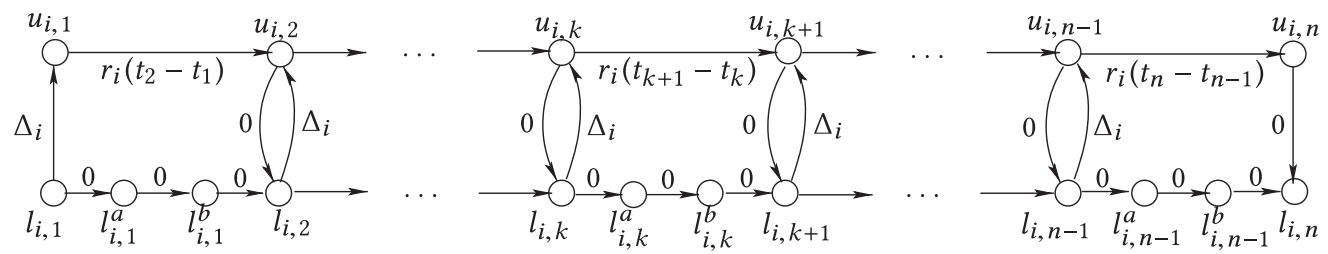

Fig. 1. The component $C_{i}$ for server $S_{i}$.
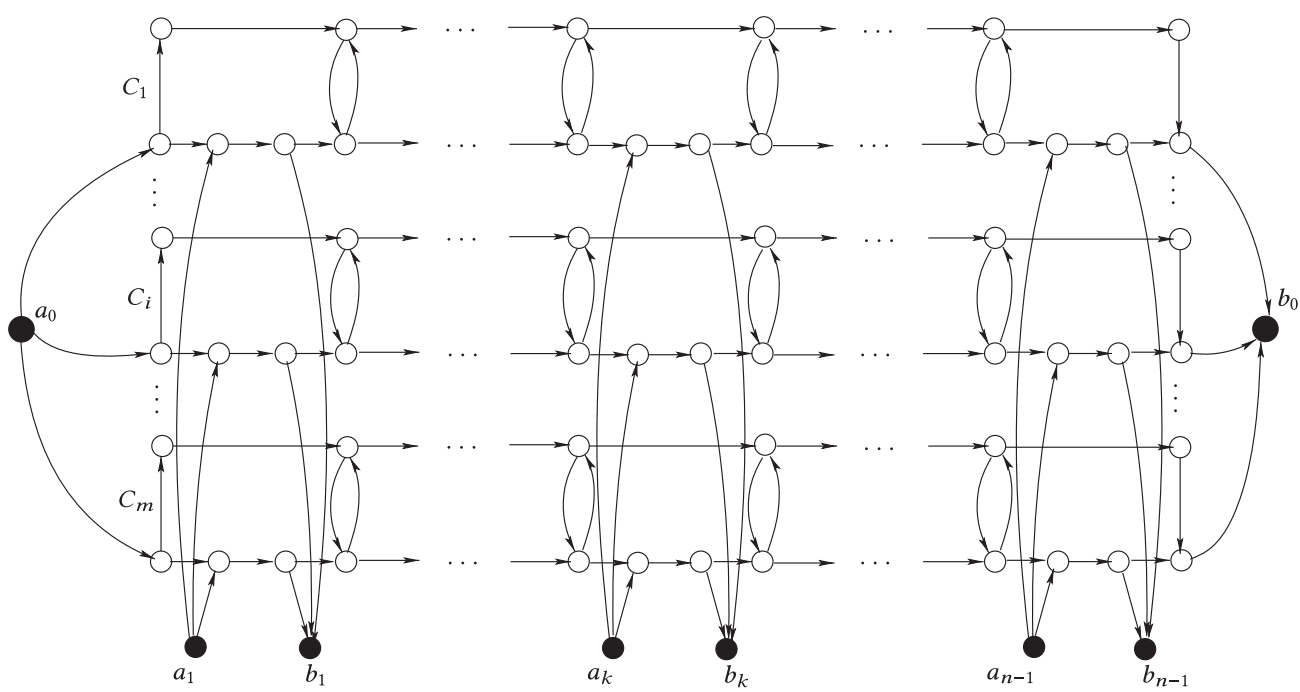

Fig. 2. The network $\mathcal{N}(\mathcal{I})$.

computing demands change at the times $t_{1}<\cdots<t_{n}$ in $T$. For any $t_{k}, 1 \leq k \leq n$, there is a vertex $u_{i, k}$ on the upper path. Vertices $u_{i, k}$ and $u_{i, k+1}$ are connected by a directed edge $\left(u_{i, k}, u_{i, k+1}\right)$ of cost $r_{i}\left(t_{k+1}-t_{k}\right), 1 \leq k \leq n-1$. This cost is equal to the energy consumed if $S_{i}$ is in the active state during $\left[t_{k}, t_{k+1}\right)$. Similarly, for any $t_{k}, 1 \leq k \leq n$, there is a vertex $l_{i, k}$ on the lower path. To ensure that at least $d_{k}$ servers are in the active state during $\left[t_{k}, t_{k+1}\right)$, if $k<n$, we need two auxiliary vertices $l_{i, k}^{a}$ and $l_{i, k}^{b}$. These vertices are again connected by directed edges. There is an edge $\left(l_{i, k}, l_{i, k}^{a}\right)$, followed by two edges $\left(l_{i, k}^{a}, l_{i, k}^{b}\right)$ and $\left(l_{i, k}^{b}, l_{i, k+1}\right)$, for any $k$ with $1 \leq k \leq n-1$. The cost of each of these edges is 0 because the energy consumption in the sleep state is 0 .

The lower and the upper path are connected by additional edges that model state transitions. Recall that all servers are in the sleep state at times $t_{1}$ and $t_{n}$. For any $k$ with $1 \leq k \leq n-1$, there is a directed edge $\left(l_{i, k}, u_{i, k}\right)$ of cost $\Delta_{i}$, representing a power-up operation of $S_{i}$ at time $t_{k}$. For any $k$ with $1<k \leq n$, there is a directed edge $\left(u_{i, k}, l_{i, k}\right)$ of cost 0 , modeling a power-down operation of $S_{i}$ at time $t_{k}$. The capacity of each edge of $C_{i}$ is equal to 1 .

The entire network. In network $\mathcal{N}(\mathcal{I})$, the components $C_{1}, \ldots, C_{m}$ are aligned in parallel and connected to a source $a_{0}$ and a sink $b_{0}$. The general structure of $\mathcal{N}(\mathcal{I})$ is depicted in Figure 2. There is a directed edge from $a_{0}$ to $l_{i, 1}$ in $C_{i}$, for any $1 \leq i \leq m$. Furthermore, there is a directed edge from $l_{i, n}$ to $b_{0}$, for any $1 \leq i \leq m$. Each of these edges has a cost of 0 and a capacity of 1 . Vertex $a_{0}$ has a supply of $m$, and $b_{0}$ has a demand of $m$. Hence, $m$ units of flow must be shipped through $C_{1}, \ldots, C_{m}$. Considering that all edges have a capacity of 1 , one unit of flow must be routed through each $C_{i}$, $1 \leq i \leq m$. Whenever the unit traverses the upper path, $S_{i}$ is in the active state. Whenever the unit traverses the lower path, $S_{i}$ is in the sleep state. 
To ensure that at least $d_{k}$ servers are in the active state during $\left[t_{k}, t_{k+1}\right), 1 \leq k \leq n-1$, we introduce additional sources and sinks. Network $\mathcal{N}(\mathcal{I})$ has a source $a_{k}$ and a sink $b_{k}$ with supply/demand $d_{k}$, for any $1 \leq k \leq n-1$. There is a directed edge from $a_{k}$ to $l_{i, k}^{a}$ on the lower path of each $C_{i}, 1 \leq i \leq m$. Furthermore, there is a directed edge from each $l_{i, k}^{b}$ to $b_{k}, 1 \leq i \leq m$. The cost and capacity of each of these edges is equal to 0 and 1 , respectively. Considering that $d_{k}$ flow units have to be shipped from $a_{k}$ to $b_{k}$, there must exist at least $d_{k}$ components $C_{i}$ in which the flow unit from $a_{0}$ to $b_{0}$ traverses the upper path from $u_{i, k}$ to $u_{i, k+1}$. Hence, the corresponding servers are in the active state during $\left[t_{k}, t_{k+1}\right)$. The encoding length of $\mathcal{N}(\mathcal{I})$ is polynomial in that of $\mathcal{I}$.

Lemma 3.3. Any feasible schedule $\Sigma$ in which state transitions are performed only at the times of $T$ corresponds to a feasible flow of cost $E(\Sigma)$.

Proof. Given $\Sigma$, we construct a flow as follows. One flow unit is transferred from $a_{0}$ to $b_{0}$ via component $C_{i}$, for any $1 \leq i \leq m$. Within $C_{i}$, the flow unit mimics the actions of $S_{i}$. Whenever $S_{i}$ powers up at a time $t_{k}, 1 \leq k \leq n-1$, the flow unit traverses edge $\left(l_{i, k}, u_{i, k}\right)$. If $S_{i}$ resides in the active state in $\left[t_{k}, t_{k+1}\right)$, the flow unit traverses $\left(u_{k}, u_{k+1}\right)$; otherwise, it traverses the path from $l_{k}$ to $l_{k+1}$. Whenever the server powers down at time $t_{k}, 1<k \leq n$, the flow unit takes edge $\left(u_{i, k}, l_{i, k}\right)$. The cost incurred by the flow unit in $C_{i}$ is equal to the energy consumed by $S_{i}$. Consider any $k$ with $1 \leq k \leq n-1$. Considering that $\sum$ is feasible, at least $d_{k}$ servers are in the active state in $\left[t_{k}, t_{k+1}\right)$. Hence, there are at least $d_{k}$ components $C_{i}$ in which the flow unit traverses the edge $\left(u_{k}, u_{k+1}\right)$ on the upper path. We may choose any $d_{k}$ of these components. For each such $C_{i}$, one unit of flow is shipped from $a_{k}$ to $b_{k}$ via the auxiliary vertices $l_{i, k}^{a}$ and $l_{i, k}^{b}$. Thus, $d_{k}$ flow units are transferred from $a_{k}$ to $b_{k}$ and the overall flow is feasible. The total cost of the flow is equal to $E(\Sigma)$ because the shipment of flow from any $a_{k}$ to $b_{k}, 1 \leq k \leq n-1$, does not incur cost.

\subsection{Analysis of Flows}

We analyze feasible flows in $\mathcal{N}(\mathcal{I})$. The goal is to show that any feasible flow $f$ can be converted into one that corresponds to a feasible schedule $\Sigma$ for $\mathcal{I}$; the energy consumed by $\Sigma$ will be equal to the cost of $f$. The conversion is not immediate. A feasible flow might not be well behaved, i.e., flow shipped out of a source $a_{k}$ is not necessarily routed to $b_{k}, 0 \leq k \leq n-1$. It may happen that flow leaving $a_{k}$ is routed to a sink $b_{k^{\prime}}$, where $k^{\prime}>k$, or to $b_{0}$.

In $\mathcal{N}(\mathcal{I})$, all edge capacities and supplies/demands are integer values. Hence, in $\mathcal{N}(\mathcal{I})$, there exists a minimum-cost flow that is integral. A flow $f$ is called integral if the flow $f(e)$ along any edge $e$ takes an integer value. Moreover, there exist polynomial time combinatorial algorithms that compute an integral minimum-cost flow, given a network with integer edge capacities and supplies/demands; see Ahuja et al. [1].

We will always work with a flow $f$ in $\mathcal{N}(\mathcal{I})$ that is integral. Such a flow translates into a state transition schedule for the servers if, for each $C_{i}$ and each $k$, one flow unit traverses either the upper path from $u_{i, k}$ to $u_{i, k+1}$ or the lower path from $l_{i, k}$ to $l_{i, k+1}$. Formally, we call an integral flow consistent in $\left[t_{k}, t_{k+1}\right)$, where $1 \leq k \leq n-1$, if $f\left(u_{i, k}, u_{i, k+1}\right)+f\left(l_{i, k}, l_{i, k}^{a}\right)=1$ holds for all $i=1, \ldots, m$. In this definition, we only consider flow from $l_{i, k}$ to $l_{i, k}^{a}$. This will be sufficient for our purposes. An integral flow is called consistent if it is consistent in all intervals $\left[t_{k}, t_{k+1}\right), 1 \leq$ $k \leq n-1$. In the following, we will prove that any feasible integral flow can be converted into one that is consistent. The next lemma identifies some useful properties.

Lemma 3.4. Let $f$ be any feasible integral flow in $\mathcal{N}(\mathcal{I})$.

(a) Then $f$ is consistent in $\left[t_{1}, t_{2}\right)$.

(b) Suppose that $f$ is consistent in $\left[t_{1}, t_{2}\right), \ldots,\left[t_{k}, t_{k+1}\right)$ but not in $\left[t_{k+1}, t_{k+2}\right)$. Then there exist $C_{i}$ and $C_{j}$ such that $f\left(u_{i, k+1}, u_{i, k+2}\right)+f\left(l_{i, k+1}, l_{i, k+1}^{a}\right)=2$ and $f\left(u_{j, k+1}, u_{j, k+2}\right)+f\left(l_{j, k+1}\right.$, 


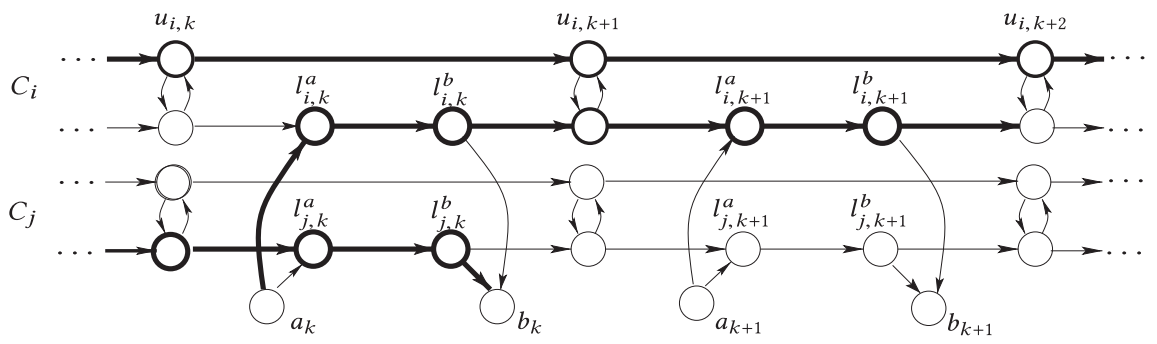

Fig. 3. A flow that is not consistent in $\left[t_{k+1}, t_{k+2}\right)$.

$\left.l_{j, k+1}^{a}\right)=0$, cf. Figure 3. In $C_{i}$, flow $f$ satisfies $f\left(u_{i, k}, u_{i, k+1}\right)=1$. One flow unit is shipped from source $a_{k}$ to $l_{i, k}^{a}$ and is further routed to $l_{i, k+1}$. In $C_{j}$, flow $f$ satisfies $f\left(l_{j, k}, l_{j, k}^{a}\right)=1$. This unit is routed via $l_{j, k}^{b}$ to sink $b_{k}$.

Proof. For the proof of part (a), we observe that a supply of $m$ is shipped from $a_{0}$ to the components $C_{1}, \ldots, C_{m}$. Considering that all edge capacities are 1 , exactly one unit of flow enters $l_{i, 1}$ in $C_{i}, 1 \leq i \leq m$. The unit can traverse the edge to $l_{i, 1}^{a}$. Alternatively, it can move to the upper path of $C_{i}$ and traverse $\left(u_{i, 1}, u_{i, 2}\right)$. In addition, considering that further flow can be injected into $C_{i}$ only from a source $a_{k}$ reaching vertex $l_{i, k}^{a}, 1 \leq k \leq n-1$, flow $f$ is consistent in $\left[t_{1}, t_{2}\right)$.

We next prove part (b). Flow $f$ is consistent in $\left[t_{1}, t_{2}\right), \ldots,\left[t_{k}, t_{k+1}\right)$. Considering the last of these intervals and all $C_{1}, \ldots, C_{m}$, we obtain $\sum_{i=1}^{m}\left(f\left(u_{i, k}, u_{i, k+1}\right)+f\left(l_{i, k}, l_{i, k}^{a}\right)\right)=m$. Source $a_{k}$ injects a total of $d_{k}$ flow units into $C_{1}, \ldots, C_{m}$, but the same amount is also absorbed by sink $b_{k}$. Hence, exactly $m$ flow units traverse the set of edges $\left(u_{i, k+1}, u_{i, k+2}\right)$ and $\left(l_{i, k+1}, l_{i, k+1}^{a}\right), 1 \leq i \leq m$. Formally, $\sum_{i=1}^{m}\left(f\left(u_{i, k+1}, u_{i, k+2}\right)+f\left(l_{i, k+1}, l_{i, k+1}^{a}\right)\right)=m$. Considering that $f$ is not consistent in $\left[t_{k+1}, t_{k+2}\right)$, then there must exist $C_{i}$ and $C_{j}$ such that $f\left(u_{i, k+1}, u_{i, k+2}\right)+f\left(l_{i, k+1}, l_{i, k+1}^{a}\right)=2$ and $f\left(u_{j, k+1}, u_{j, k+2}\right)+f\left(l_{j, k+1}, l_{j, k+1}^{a}\right)=0$; see Figure 3. In $C_{i}$, a total of two flow units leave $u_{i, k+1}$ and $l_{i, k+1}$ along the upper and lower paths, respectively. The second flow unit must be injected from $a_{k}$ via $l_{i, k}^{a}$. The unit is further routed to $l_{i, k}^{b}$ and $l_{i, k+1}$. This also implies that $f\left(u_{i, k}, u_{i, k+1}\right)=1$ because $f$ is consistent in $\left[t_{k}, t_{k+1}\right)$. In $C_{j}$, the flow unit present along $\left(u_{j, k}, u_{j, k+1}\right)$ or $\left(l_{j, k}, l_{j, k}^{a}\right)$ can only leave the component via $l_{j, k}^{b}$ by traversing the edge to the sink $b_{k}$. This implies that $f\left(l_{j, k}, l_{j, k}^{a}\right)=1$.

\subsection{Making a Flow Consistent}

Let $f$ be a feasible integral flow in $\mathcal{N}(\mathcal{I})$. We describe how algorithm $\mathcal{A}_{1}$ modifies $f$ so that the resulting flow is consistent. By Lemma 3.4(a), $f$ is consistent in $\left[t_{1}, t_{2}\right)$. Suppose that $f$ is consistent in $\left[t_{1}, t_{2}\right), \ldots,\left[t_{k}, t_{k+1}\right)$ but not in $\left[t_{k+1}, t_{k+2}\right)$. $\mathcal{A}_{1}$ modifies the flow so that it becomes consistent in $\left[t_{1}, t_{2}\right), \ldots,\left[t_{k+1}, t_{k+2}\right)$. The modifications are performed sequentially for all further intervals.

Modifying flow. By assumption, $f$ is consistent in $\left[t_{1}, t_{2}\right), \ldots,\left[t_{k}, t_{k+1}\right)$ but not in $\left[t_{k+1}, t_{k+2}\right)$. Hence, there must exist components $C_{i}$ and $C_{j}$ with the properties specified in Lemma 3.4(b); see Figure 3. In $C_{i}$, a total of two flow units leave $u_{i, k+1}$ and $l_{i, k+1}$ along the upper and lower paths, respectively. On the upper path, one flow unit traverses the edge from $u_{i, k}$ and $u_{i, k+1}$. On the lower path, one unit is injected from $a_{k}$. This unit reaches $l_{i, k}^{a}$ and continues via $l_{i, k}^{b}$ to $l_{i, k+1}$. In $C_{j}$, no flow leaves $u_{j, k+1}$ or $l_{j, k+1}$. A flow unit is shipped from $l_{j, k}$ to $l_{j, k}^{a}$, and this unit is routed to sink $b_{k}$ via $l_{j, k}^{a}$.

As long as there exist components $C_{i}$ and $C_{j}$ as specified earlier, $\mathcal{A}_{1}$ works as follows. It determines the smallest integer $k^{\prime}$, with $k^{\prime}>k$, such that a flow unit is routed from $C_{i}$ to sink $b_{k^{\prime}}$, i.e., $f\left(l_{i, k^{\prime}}^{b}, b_{k^{\prime}}\right)=1$. Such an integer must exist, because otherwise a total of two flow units must ACM Transactions on Parallel Computing, Vol. 6, No. 3, Article 13. Publication date: October 2019. 


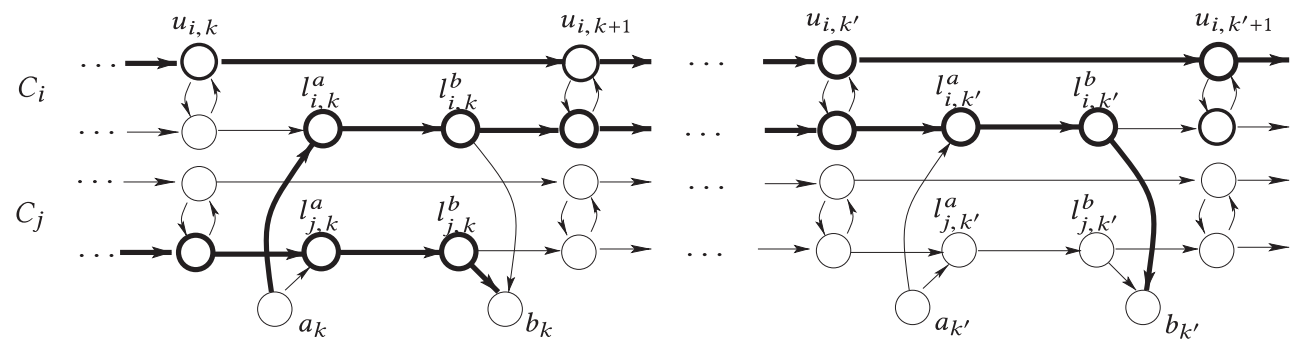

Fig. 4. No edge of $P_{j}\left(k, k^{\prime}\right)$ ships flow.

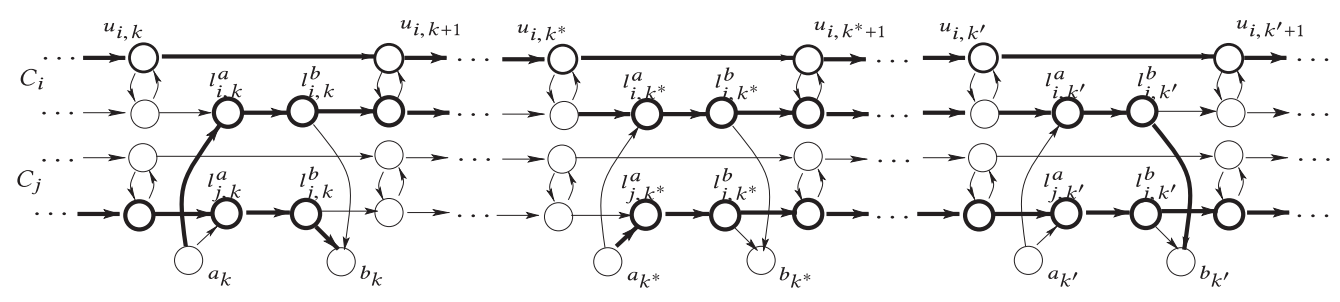

Fig. 5. Some edge of $P_{j}\left(k, k^{\prime}\right)$ ships flow.

reach the end of $C_{i}$ at $u_{i, n}$ and $l_{i, n}$. These two flow units cannot feasibly be routed to $b_{0}$ along the unit-capacity edge $\left(l_{i, n}, b_{0}\right)$. Let $P_{i}\left(k, k^{\prime}\right)$ be the path from $l_{i, k}^{b}$ to $l_{i, k^{\prime}}^{b}$ that uses only edges of the lower path of $C_{i}$. All edges of $P_{i}\left(k, k^{\prime}\right)$ carry one unit of flow. Similarly, let $P_{j}\left(k, k^{\prime}\right)$ be the path from $l_{j, k}^{b}$ to $l_{j, k^{\prime}}^{b}$ that uses only edges of the lower path of $C_{j}$. In the flow modification, there are two cases depending on whether or not $P_{j}\left(k, k^{\prime}\right)$ carries any flow.

Flow modification, type 1. Suppose that $P_{j}\left(k, k^{\prime}\right)$ does not ship any flow; see Figure 4. Loosely speaking, $\mathcal{A}_{1}$ replaces flow along $P_{i}\left(k, k^{\prime}\right)$ by flow on $P_{j}\left(k, k^{\prime}\right)$. Formally, the modified flow is as follows. In $C_{i}$, the flow unit entering $l_{i, k}^{b}$ is routed to $b_{k}$, i.e., $f^{\prime}\left(l_{i, k}^{b}, b_{k}\right)=1$. In $C_{j}$, algorithm $\mathcal{A}_{1}$ removes the flow unit leaving $l_{j, k}^{b}$, i.e., $f^{\prime}\left(l_{j, k}^{b}, b_{k}\right)=0$. For all edges $e$ of $P_{i}\left(k, k^{\prime}\right)$, the algorithm sets $f^{\prime}(e)=0$. For all edges $e$ of $P_{j}\left(k, k^{\prime}\right)$, it sets $f^{\prime}(e)=1$. Finally, it removes the flow unit leaving $l_{i, k^{\prime}}^{b}$, i.e., $f^{\prime}\left(l_{i, k^{\prime}}^{b}, b_{k^{\prime}}\right)=0$, and routes one unit from $l_{j, k^{\prime}}^{b}$ to $b_{k^{\prime}}$, i.e., $f^{\prime}\left(l_{j, k^{\prime}}^{b}, b_{k^{\prime}}\right)=1$. For all other edges not considered here, the flow remains unchanged. Obviously, after these modifications, the amount of flow routed into $b_{k}$ and $b_{k^{\prime}}$ has not changed. The flow conservation law is observed at all vertices of $P_{i}\left(k, k^{\prime}\right)$ and $P_{j}\left(k, k^{\prime}\right)$. Hence, the new flow is feasible. Furthermore, the cost of the flow has not changed because the flow update only affects edges of cost 0 . Note that $f^{\prime}\left(l_{i, k+1}, l_{i, k+1}^{a}\right)=0$ and $f^{\prime}\left(l_{j, k+1}, l_{j, k+1}^{a}\right)=1$. Hence, restricted to $C_{i}$ and $C_{j}$, the new flow is consistent in $\left[t_{k+1}, t_{k+2}\right)$.

Flow modification, type 2. Assume that some edge of $P_{j}\left(k, k^{\prime}\right)$ carries flow; see Figure 5 . Then this flow must enter $C_{j}$ from some source among $a_{k+1}, \ldots, a_{k^{\prime}} \cdot \mathcal{A}_{1}$ determines the smallest integer $k^{*}$, with $k^{*}>k$, such that $f\left(a_{k^{*}}, l_{j, k^{*}}^{a}\right)=1$. Then $k^{*} \leq k^{\prime}$. In component $C_{i}$, the corresponding edge $\left(a_{k^{*}}, l_{i, k^{*}}^{a}\right)$ does not ship flow because all edges of $P_{i}\left(k, k^{\prime}\right)$ carry one unit of flow and no further unit can be injected from $a_{k^{*}}$. Let $P_{i}\left(k, k^{*}\right)$ be the path from $l_{i, k}^{b}$ to $l_{i, k^{*}}^{a}$ that uses only edges of the lower path of $C_{i}$. Analogously, let $P_{j}\left(k, k^{*}\right)$ be the path from $l_{j, k}^{b}$ to $l_{j, k^{*}}^{a}$ that uses only edges of the lower path of $C_{j}$. $\mathcal{A}_{1}$ replaces flow on $P_{i}\left(k, k^{*}\right)$ by flow on $P_{j}\left(k, k^{*}\right)$. More specifically, the flow unit routed into $l_{i, k}$ is shipped to $b_{k}$, i.e., $f^{\prime}\left(l_{i, k}^{b}, b_{k}\right)=1$. The flow unit on edge $\left(l_{j, k}^{b}, b_{k}\right)$ is removed. For all edges $e$ of $P_{i}\left(k, k^{*}\right), \mathcal{A}_{1}$ sets $f^{\prime}(e)=0$. For all edges $e$ of $P_{j}\left(k, k^{*}\right)$, it sets $f^{\prime}(e)=1$. Finally, it sets $f^{\prime}\left(a_{k^{*}}, l_{i, k^{*}}^{a}\right)=1$ and $f^{\prime}\left(a_{k^{*}}, l_{j, k^{*}}^{a}\right)=0$. The new flow is feasible, and during the modification 
the cost has not changed. Restricted to $C_{i}$ and $C_{j}$, the new flow is consistent in $\left[t_{k+1}, t_{k+2}\right)$ because $f^{\prime}\left(l_{i, k+1}, l_{i, k+1}^{a}\right)=0$ and $f^{\prime}\left(l_{j, k+1}, l_{j, k+1}^{a}\right)=1$.

We remark that the preceding flow modifications do not change flow in components other than $C_{i}$ and $C_{j}$. By repeating the flow update operations for other pairs of network components violating consistency, $\mathcal{A}_{1}$ obtains a flow that is consistent in $\left[t_{1}, t_{2}\right), \ldots,\left[t_{k+1}, t_{k+2}\right)$. The total number of steps to perform the modifications is polynomial in $\mathcal{N}(\mathcal{I})$. The next lemma summarizes the result.

LEMMA 3.5. Let $f$ be a feasible integral flow of cost $C$ in $\mathcal{N}(\mathcal{I})$. Then $f$ can be transformed into a feasible integral flow that is consistent and has cost $C$. The transformation takes a polynomial number of steps.

\subsection{Establishing the Theorem}

We show an important property of feasible consistent flows in $\mathcal{N}(\mathcal{I})$.

Lemma 3.6. In any feasible integral flow $f$ that is consistent, all flow leaving $a_{k}$ is routed to $b_{k}$, $1 \leq k \leq n-1$.

Proof. Fix a $k$ with $1 \leq k \leq n-1$. Consider a unit of flow that leaves $a_{k}$ and enters a component $C_{i}$ via $l_{i, k}^{a}$. The unit is routed to $l_{i, k}^{b}$. Considering that $f$ is consistent in $\left[t_{k}, t_{k+1}\right)$, it satisfies $f\left(u_{i, k}, u_{i, k+1}\right)+f\left(l_{i, k}, l_{i, k}^{a}\right)=1$. In addition, considering that all edge capacities are 1 , no further flow can enter $l_{i, k}^{a}$. Hence, $f\left(u_{i, k}, u_{i, k+1}\right)=1$. First assume that $k<n-1$. In this case, the flow unit on $\left(u_{i, k}, u_{i, k+1}\right)$ will be routed on either $\left(u_{i, k+1}, u_{i, k+2}\right)$ or $\left(l_{i, k+1}, l_{i, k+1}^{a}\right)$. As $f$ is consistent in $\left[t_{k+1}, t_{k+2}\right)$, the flow unit entering $l_{i, k}^{b}$ must be shipped to $b_{k}$ and cannot be sent to $l_{i, k+1}$. If $k=n-1$, then the flow unit on $\left(u_{i, k}, u_{i, k+1}\right)$ is routed to $l_{i, n}$ and $b_{0}$. Considering that all edge capacities are 1 , no further flow can be shipped on $\left(l_{i, n}, b_{0}\right)$. Again, the flow unit entering $l_{i, k}^{b}$ must be routed to $b_{k}$ and cannot be sent to $l_{i, k+1}$.

We finish the proof of Theorem 3.1. Given problem instance $\mathcal{I}, \mathcal{A}_{1}$ constructs $\mathcal{N}(\mathcal{I})$ and computes an integral minimum-cost flow $f^{*}$ using a combinatorial algorithm. Executing the flow modifications described earlier, the algorithm obtains an integral minimum-cost flow $f$ that is consistent. Lemma 3.6 implies that in $f$, all flow units leaving $a_{0}$ are transferred to $b_{0}$. By the edge capacity constraints, one unit of flow is transferred through each $C_{i}, 1 \leq i \leq m$. $\mathcal{A}_{1}$ derives a schedule $\Sigma$ for $I$ by keeping track of these flow units in $C_{1}, \ldots, C_{m}$. Consider component $C_{i}, 1 \leq i \leq m$. While the flow unit traverses the upper path, server $S_{i}$ is in the active state. While the flow unit traverses the lower path, $S_{i}$ is in the sleep state. If the flow traverses an edge $\left(l_{i, k}, u_{i, k}\right), S_{i}$ powers up at time $t_{k}$. If the flow traverses $\left(u_{i, k}, l_{i, k}\right)$, the server powers down at time $t_{k}$. The energy consumed by $S_{i}$ is exactly equal to the cost incurred by the flow unit traversing $C_{i}$. Hence, the energy consumed by $\Sigma$ is equal to the cost of $f$, and this is equal to the cost of $f^{*}$.

It remains to verify that $\Sigma$ is feasible. By Lemma 3.6, in $f$ all flow units leaving $a_{k}$ are shipped to $b_{k}, 1 \leq k \leq n-1$. Consider any fixed $k, 1 \leq k \leq n-1$. There must exist $d_{k}$ components $C_{i}$ such that a flow unit is routed from $a_{k}$ to $l_{i, k}^{a}$ and further on to $l_{i, k}^{b}$ and $b_{k}$. Considering that $f$ is consistent in $\left[t_{k}, t_{k+1}\right)$, it satisfies $f\left(u_{i, k}, u_{i, k+1}\right)=1$. If $f\left(l_{i, k}, l_{i, k}^{a}\right)=1$, then two units of flow would leave $l_{i, k}^{a}$, violating the capacity of the outgoing edge. Thus, in $\left[t_{k}, t_{k+1}\right)$, at least $d_{k}$ servers are in the active state. Optimality of $\Sigma$ follows from Proposition 2.2 and Lemma 3.3.

\section{SERVERS WITH MULTIPLE STATES}

We develop an approximation algorithm for DPM in the general setting that each server may have an arbitrary number of states. Let $\mathcal{I}=(\mathcal{S}, \mathcal{D})$ be an input with $\tau$ server types, i.e., each server of $\mathcal{S}$ belongs to one of $\tau$ classes, where $\tau \in \mathbb{N}$. Formally, $\mathcal{S}$ is partitioned into $\mathcal{S}_{1}, \ldots, \mathcal{S}_{\tau}$. Within 


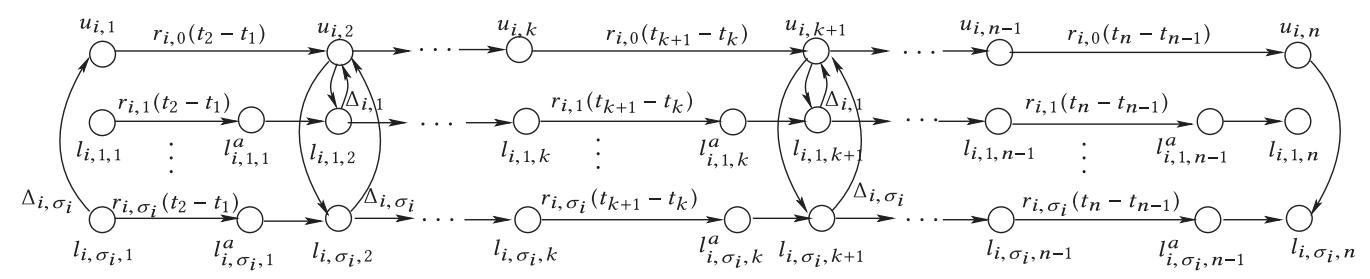

Fig. 6. The component $C_{i}$ for server type $i$. Unlabeled edges connecting two vertices have cost 0 .

each server type/class $\mathcal{S}_{i}, 1 \leq i \leq \tau$, all servers are identical. Every server of $\mathcal{S}_{i}$ has $\sigma_{i}+1$ states $s_{i, 0}, \ldots, s_{i, \sigma_{i}}$ with power consumption rates $r_{i, 0}>\cdots>r_{i, \sigma_{i}}$. Here, $s_{i, 0}$ is again the active state; the other states are low-power modes. The energy needed to transition from $s_{i, j}$ to $s_{i, 0}$ is denoted by $\Delta_{i, j}, 1 \leq j \leq \sigma_{i}$. The state transition energy from the active state to any lower-power state is 0 . The servers of $\mathcal{S}_{i}$ are independent and not synchronized. Over the time horizon, each server may reside in individual states and perform state transitions independent of the other servers. Let $m_{i}$ be the number of servers in $\mathcal{S}_{i}$. Then $\sum_{i=1}^{\tau} m_{i}=m$.

TheOREM 4.1. Let $\mathcal{I}$ be an instance of DPM with $\tau$ server types. A schedule whose energy consumption is at most $\tau$ times the minimum one for $I$ can be computed in polynomial time based on a minimum-cost two-commodity flow computation.

In the remainder of this section, we develop an algorithm $\mathcal{A}_{2}$ that, given $\mathcal{I}=(\mathcal{S}, \mathcal{D})$, constructs a feasible schedule attaining a $\tau$-approximation to the optimal energy consumption. By Proposition 2.2, we restrict ourselves to schedules with the following two properties. (1) While a server is in low-power mode, it uses a single state. (2) State transitions are performed only at the times of $T$.

Algorithm $\mathcal{A}_{2}$ constructs a network $\mathcal{N}(\mathcal{I})$. Compared to the construction in Section 3 , the main differences are as follows. Each network component will represent a class of servers so that the encoding length of $\mathcal{N}(\mathcal{I})$ is polynomial in that of $\mathcal{I}$. A component has a collection of lower paths corresponding to the various low-power modes of the servers. We need a second commodity to ensure that computing demands are met. This will allow us to reduce the number of auxiliary vertices on the lower paths. We will explain the need of the second commodity later, before Lemma 4.2.

Any schedule $\Sigma$ as specified in Proposition 2.2 corresponds to a feasible flow of cost $E(\Sigma)$ in $\mathcal{N}(\mathcal{I})$. Given $\mathcal{N}(\mathcal{I}), \mathcal{A}_{2}$ computes a minimum-cost flow $f^{*}$. Considering that the network involves two commodities, $f^{*}$ is not integral but fractional in general. In a sequence of rounding and packing operations, $\mathcal{A}_{2}$ transforms $f^{*}$ into an integral flow that guides the construction of a feasible schedule for $\mathcal{I}$. The cost of the integral flow and the constructed schedule will be at most $\tau$ times that of $f^{*}$.

\subsection{Construction of the Network}

We describe $\mathcal{N}(\mathcal{I})$, given $\mathcal{I}=(\mathcal{S}, \mathcal{D})$.

Network components with multiple paths. For each server type $i$, the network contains a component $C_{i}, 1 \leq i \leq \tau$. The component represents all $m_{i}$ servers of $\mathcal{S}_{i}$. Exactly $m_{i}$ flow units will be routed through $C_{i}$, modeling the individual states and actions of the servers. Component $C_{i}$ consists of an upper path and $\sigma_{i}$ lower paths. The general structure is depicted in Figure 6. We search for an optimal schedule in which state transitions are performed only at times $t_{1}<\cdots<t_{n}$ in $T$, cf. Proposition 2.2. In $C_{i}$, the upper path corresponds to the active state of the servers of $\mathcal{S}_{i}$. For any $t_{k}, 1 \leq k \leq n$, there is a vertex $u_{i, k}$ on the upper path. Vertices $u_{i, k}$ and $u_{i, k+1}$ are connected by a directed edge $\left(u_{i, k}, u_{i, k+1}\right)$ of cost $r_{i, 0}\left(t_{k+1}-t_{k}\right), 1 \leq k \leq n-1$. The cost is equal to energy 


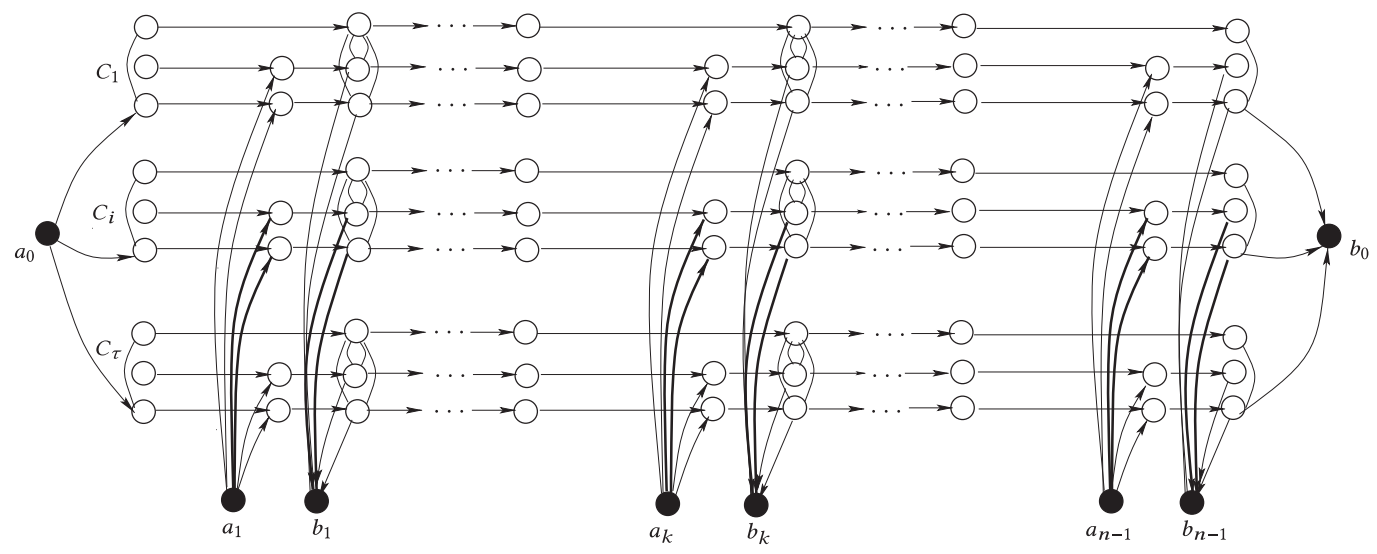

Fig. 7. The network $\mathcal{N}(\mathcal{I})$. To maintain the readability of the figure, for $a_{k}$ and $b_{k}$ with $1 \leq k \leq n-1$, only the links into $C_{i}$ are shown as solid lines. For the other components, the links are shown as dashed lines.

consumed by one server of $\mathcal{S}_{i}$ if it resides in the active state during $\left[t_{k}, t_{k+1}\right)$. The capacity of $\left(u_{i, k}, u_{i, k+1}\right)$, and in fact of all edges of $C_{i}$, is equal to $m_{i}$, reflecting that $C_{i}$ represents $m_{i}$ servers in $\mathcal{S}_{i}$.

The $\sigma_{i}$ lower paths correspond to the $\sigma_{i}$ low-power states. Consider any $j$ with $1 \leq j \leq \sigma_{i}$. On lower path $j$, there is a vertex $l_{i, j, k}$ and an auxiliary vertex $l_{i, j, k}^{a}$, for any $1 \leq k \leq n-1$. Moreover, there is a final vertex $l_{i, j, n}$. The auxiliary vertices will help to ensure that a total of at least $d_{k}$ flow units traverse the edges $\left(u_{i, k}, u_{i, k+1}\right)$ on the upper paths, considering all of the components $C_{i}$, $1 \leq i \leq \tau$. We do not need a second auxiliary vertex $l_{i, j, k}^{b}$ because we work with two commodities. On lower path $j$, the vertices are connected as follows. For any $k$, where $1 \leq k \leq n-1$, there is a directed edge $\left(l_{i, j, k}, l_{i, j, k}^{a}\right)$ of cost $r_{i, j}\left(t_{k+1}-t_{k}\right)$, representing the energy consumed by a server if it is in state $s_{i, j}$ during $\left[t_{k}, t_{k+1}\right)$. Furthermore, there is an edge $\left(l_{i, j, k}^{a}, l_{i, j, k+1}\right)$ of cost 0 .

The upper path is connected to the lower paths by additional edges that model state transitions. We assume that at times $t_{1}$ and $t_{n}$, all servers of $\mathcal{S}_{i}$ are in the deepest low-power state $s_{i, \sigma_{i}}$. Thus, there is a directed edge $\left(l_{i, \sigma_{i}, 1}, u_{i, 1}\right)$ of cost $\Delta_{i, \sigma_{i}}$ modeling possible power-up operations of servers at time $t_{1}$. Furthermore, there is a directed edge $\left(u_{i, n}, l_{i, \sigma_{i}, n}\right)$ of cost 0 representing power-down operations at time $t_{n}$. For any $1<k<n$ and any $1 \leq j \leq \sigma_{i}$, there is a directed edge $\left(u_{i, k}, l_{i, j, k}\right)$ of cost 0 and a directed edge $\left(l_{i, j, k}, u_{i, k}\right)$ of cost $\Delta_{i, j}$. As we consider schedules specified in Proposition 2.1, there are no state transitions among low-power states; thus, there are no edges between the lower paths. (We remark that on lower path $j, 1 \leq j<\sigma_{i}$, we could remove the first and the last vertex, but it is not important.) Note again that the capacity of each edge of $C_{i}$ is $m_{i}$.

The network with two commodities. In $\mathcal{N}(\mathcal{I})$, components $C_{1}, \ldots, C_{\tau}$ are aligned in parallel and connected to vertices $a_{0}$ and $b_{0}$, cf. Figure 7. These two vertices inject and absorb flow of commodity 1 . Specifically, $a_{0}$ has a supply of $m$ and $b_{0}$ has a demand of $m$ of commodity 1 . The connections are as follows. At times $t_{1}$ and $t_{n}$, the servers are in the deepest low-power mode. Hence, for any $1 \leq i \leq \tau$, there exist directed edges $\left(a_{0}, l_{i, \sigma_{i}, 1}\right)$ and $\left(l_{i, \sigma_{i}, n}, b_{0}\right)$. Each of these edges has a cost of 0 and a capacity of $m_{i}$ so that $m_{i}$ flow units can be routed from $a_{0}$ to $b_{0}$ via $C_{i}$.

Network $\mathcal{N}(\mathcal{I})$ contains further sources and sinks that inject and absorb flow of commodity 2 . This second commodity will ensure that the computing demands are met. Consider any $k$ with $1 \leq k \leq n-1$. There is a source $a_{k}$ and a sink $b_{k}$ with a supply/demand of

$$
D_{k}=\sum_{i=1}^{\tau} m_{i}\left(\sigma_{i}-1\right)+d_{k}
$$




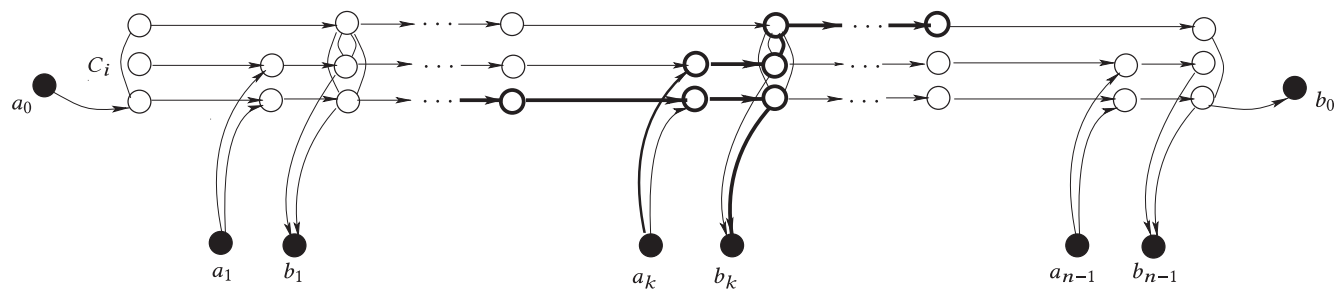

Fig. 8. Feasible flow and state changes with a single commodity.

of commodity 2. Vertices $a_{k}$ and $b_{k}$ are connected to all lower paths in the components. For any $i$ and $j$ with $1 \leq i \leq \tau$ and $1 \leq j \leq \sigma_{i}$, there is a directed edge $\left(a_{k}, l_{i, j, k}^{a}\right)$ into the auxiliary vertex on lower path $j$ in $C_{i}$. Moreover, there is a directed edge $\left(l_{i, j, k+1}, b_{k}\right)$ from the following vertex on the lower path into $b_{k}$. Each of these edges has a cost of 0 and a capacity of $m_{i}$. Lemma 4.2 states that in any feasible flow, for any $1 \leq k \leq n-1$, at least $d_{k}$ units will be shipped along the edges $\left(u_{i, k}, u_{i, k+1}\right)$ on the upper paths of the components $C_{1}, \ldots, C_{\tau}$.

So far, we have specified the total capacity of any edge in $\mathcal{N}(\mathcal{I})$. It remains to specify edge capacity constraints for the two commodities. Consider any $1 \leq i \leq \tau$. For any edge of $C_{i}$, the capacity of commodity 1 is $m_{i}$. The same holds true for the edges $\left(a_{0}, l_{i, \sigma_{i}, 1}\right)$ and $\left(l_{i, \sigma_{i}, n}, b_{0}\right)$. On all edges leaving $a_{k}$ or entering $b_{k}, 1 \leq k \leq n-1$, the capacity of commodity 1 is equal to 0 . Hence, flow of commodity 1 must not traverse these edges. In the network components, commodity 2 may only traverse the edges from the auxiliary vertices to the subsequent vertices on the lower paths. Hence, commodity 2 has a capacity constraint of $m_{i}$ on each of the edges $\left(a_{k}, l_{i, j, k}^{a}\right),\left(l_{i, j, k}^{a}, l_{i, j, k+1}\right)$ and $\left(l_{i, j, k+1}, b_{k}\right)$, where $1 \leq i \leq \tau, 1 \leq j \leq \sigma_{i}$ and $1 \leq k \leq n-1$. For all other edges in $\mathcal{N}(\mathcal{I})$, commodity 2 has a capacity of 0 . Hence, all flow from $a_{k}, 1 \leq k \leq n-1$, must be shipped to $b_{k}$ via edges $\left(l_{i, j, k}^{a}, l_{i, j, k+1}\right)$. The encoding length of $\mathcal{N}(\mathcal{I})$ is polynomial in that of $\mathcal{I}$ because the $m_{i}$ identical servers of $\mathcal{S}_{i}$ are modeled by a single component $C_{i}, 1 \leq i \leq \tau$.

We explain why in $\mathcal{N}(\mathcal{I})$ two commodities are indeed essential. Consider the network as described earlier but with a single commodity only. In this case, a feasible flow can change among low-power states at no cost and a minimum-cost flow does not correspond to a feasible schedule with the same energy consumption. More specifically, consider the situation depicted in Figure 8, where we focus on a network component $C_{i}$ in the time interval $\left[t_{k}, t_{k+1}\right)$. Suppose for simplicity that $C_{i}$ represents a single server. This server resides in the deepest sleep state prior to $t_{k}$ and also during $\left[t_{k}, t_{k+1}\right)$. Now, a flow unit is injected from $a_{k}$ into the path representing the highest sleep state $s_{i, 1}$. This flow unit continues on to the active state, whereas the flow unit along the lowest path is shipped to $b_{k}$. Thus, at time $t_{k+1}$, the server powers up to the active state but at a reduced cost smaller than $\Delta_{i, \sigma_{i}}$. In general, with a single commodity, state changes, particularly power-up operations, can be performed without accurate payments.

We proceed with the analysis of the network $\mathcal{N}(\mathcal{I})$, incorporating two commodities, as specified earlier. Lemma 4.2, as mentioned, identifies an important property of feasible flow.

LEMmA 4.2. In $\mathcal{N}(\mathcal{I})$, there exists a feasible flow. Any feasible flow $f$ satisfies $\sum_{i=1}^{\tau} f\left(u_{i, k}, u_{i, k+1}\right) \geq$ $d_{k}$, for $1 \leq k \leq n-1$.

Proof. A feasible flow can easily be constructed as follows. For any $i, 1 \leq i \leq \tau$, exactly $m_{i}$ flow units of commodity 1 are shipped from $a_{0}$ to $b_{0}$ along the upper path of $C_{i}$. When entering $C_{i}$ at $l_{i, \sigma_{i}, 1}$ the flow units move directly to the upper path. When reaching the end of $C_{i}$ at $u_{i, 0, n}$, the flow is routed to $l_{i, \sigma_{i}, n}$. Consider any $1 \leq k \leq n-1$. No flow of commodity 1 is routed along the edges $\left(l_{i, j, k}^{a}, l_{i, j, k+1}\right)$ on the lower paths, where $1 \leq i \leq \tau$ and $1 \leq j \leq \sigma_{i}$. Hence, the total residual 
capacity of these edges is $\sum_{i=1}^{\tau} m_{i} \sigma_{i}=\sum_{i=1}^{\tau} m_{i}\left(\sigma_{i}-1\right)+m \geq D_{k}$; see (1) for the definition of $D_{k}$. We conclude that the supply of commodity 2 at $a_{k}$ can be shipped in an arbitrary way to $b_{k}$ along the edges of the lower paths. We only have to observe that the capacity of $\left(l_{i, j, k}^{a}, l_{i, j, k+1}\right)$ is $m_{i}$, which is equal to the capacity of the edges from $a_{k}$ to $l_{i, j, k}^{a}$ and from $l_{i, j, k+1}$ to $b_{k}$.

Let $f$ be any feasible flow and consider any $k$ with $1 \leq k \leq n-1$. On the upper paths of the network components, there is a collection of edges $\left(u_{i, k}, u_{i, k+1}\right), 1 \leq i \leq \tau$. On the lower paths, there is a collection of edges $\left(l_{i, j, k}^{a}, l_{i, j, k+1}\right)$, where $1 \leq i \leq \tau$ and $1 \leq j \leq \sigma_{i}$. Together, these edges must route $\sum_{i=1}^{\tau} m_{i}$ units of commodity 1 and $D_{k}=\sum_{i=1}^{\tau} m_{i}\left(\sigma_{i}-1\right)+d_{k}$ units of commodity 2 . Thus, the total flow along all of these edges is $\sum_{i=1}^{\tau} m_{i} \sigma_{i}+d_{k}$. The total capacity of the edges $\left(l_{i, j, k}^{a}, l_{i, j, k+1}\right)$, where $1 \leq i \leq \tau$ and $1 \leq j \leq \sigma_{i}$, is $\sum_{i=1}^{\tau} m_{i} \sigma_{i}$. Hence, at least $d_{k}$ units of flow must be shipped along the edges of the upper paths.

The following lemma states that every optimal schedule with the properties of Proposition 2.2 translates to a feasible flow with the same energy/cost.

Lemma 4.3. Let $\Sigma$ be an optimal schedule as specified in Proposition 2.2. Then $\Sigma$ corresponds to a feasible flow of cost $E(\Sigma)$ in $\mathcal{N}(\mathcal{I})$.

Proof. Given $\Sigma$, we construct a feasible flow. Consider any server type $i$ with set $\mathcal{S}_{i}, 1 \leq i \leq \tau$. Exactly $m_{i}$ units of commodity 1 are shipped from $a_{0}$ to $b_{0}$ via component $C_{i}$. For each server $S \in \mathcal{S}_{i}$, one flow unit is routed, modeling the states and actions of $S$. If $S$ transitions from low-power state $s_{i, j}, 1 \leq j \leq \sigma_{i}$, to the active state at time $t_{k}$, the flow unit traverses $\left(l_{i, j, k}, u_{i, k}\right)$. Conversely, if $S$ moves from the active state to low-power state $s_{i, j}$ at time $t_{k}$, the flow unit traverses $\left(u_{i, k}, l_{i, j, k}\right)$. If $S$ resides in the active state during $\left[t_{k}, t_{k+1}\right)$, the flow unit traverses $\left(u_{i, k}, u_{i, k+1}\right)$ on the upper path. If $S$ is in state $s_{i, j}$ during $\left[t_{k}, t_{k+1}\right)$, the flow unit traverses lower path $j$ from $l_{i, j, k}$ to $l_{i, j, k+1}$, where $1 \leq j \leq \sigma_{i}$. All edge capacities are observed because all edges of $C_{i}$, as well as edges $\left(a_{0}, l_{i, \sigma_{i}, 1}\right)$ and $\left(l_{i, \sigma_{i}, n}, b_{0}\right)$, have a capacity of $m_{i}$, for commodity 1 and globally.

It remains to route the flow of commodity 2. Consider any $k, 1 \leq k \leq n-1$. Considering that $\sum$ is feasible, at least $d_{k}$ servers of $\mathcal{S}$ reside in the active state during $\left[t_{k}, t_{k+1}\right)$. Thus, a total of at least $d_{k}$ flow units traverse the edges $\left(u_{i, k}, u_{i, k+1}\right)$ on the upper paths of the components $C_{i}, 1 \leq i \leq \tau$. This implies that the total residual capacity of the edges $\left(l_{i, j, k}^{a}, l_{i, j, k+1}\right)$, where $1 \leq i \leq \tau$ and $1 \leq j \leq \sigma_{i}$, is at least $\sum_{i=1}^{\tau} m_{i} \sigma_{i}-\left(\sum_{i=1}^{\tau} m_{i}-d_{k}\right)$. The first term corresponds to the total capacity of the edges $\left(l_{i, j, k}^{a}, l_{i, j, k+1}\right)$, where $1 \leq i \leq \tau$ and $1 \leq j \leq \sigma_{i}$. The second term is an upper bound on the flow of commodity 1 shipped along these edges. Hence, the residual capacity is at least $D_{k}$, cf. (1). The capacity of $\left(a_{k}, l_{i, j, k}^{a}\right)$ and $\left(l_{i, j, k+1}, b_{k}\right)$ is not smaller than the residual capacity of $\left(l_{i, j, k}^{a}, l_{i, j, k+1}\right)$, for any $1 \leq i \leq \tau$ and $1 \leq j \leq \sigma_{i}$. Thus, the supply of $D_{k}$ of commodity 2 can feasibly be routed to $b_{k}$. The cost of the flow of commodity 1 is exactly $E(\Sigma)$. The cost incurred by commodity 2 is 0 because this flow only traverses edges of cost 0 .

\subsection{Outline of the Algorithm and Flow Properties}

Given $\mathcal{N}(\mathcal{I})$, algorithm $\mathcal{A}_{2}$ computes a feasible minimum-cost flow $f^{*}$. By Lemma 4.3, an optimal schedule with the properties of Proposition 2.2 corresponds to a feasible flow with the same cost. Thus, the cost of $f^{*}$, denoted by $\cos t\left(f^{*}\right)$, is a lower bound on the energy consumed by an optimal schedule for $\mathcal{I}$. Considering that $f^{*}$ involves two commodities, it is fractional in general. In particular, it may be fractional on the upper paths of the components. On the corresponding edges the flow has to be raised, for sufficiently many components, so that a feasible schedule for $\mathcal{I}$ can be derived later. $\mathcal{A}_{2}$ modifies $f^{*}$ in three main steps. The resulting flow is integral. (1) First, $f^{*}$ is scaled by a factor of $\tau$. (2) The scaled flow is modified so that it becomes integral on the upper 
paths of the components. Specifically, on edge $\left(u_{i, k}, u_{i, k+1}\right)$, exactly

$$
d_{i, k}=\min \left\{m_{i},\left\lfloor\tau f^{*}\left(u_{i, k}, u_{i, k+1}\right)\right\rfloor\right\}
$$

units of flow are routed, where $1 \leq i \leq \tau$ and $1 \leq k \leq n-1$. In Lemma 4.4, we show that $\sum_{i=1}^{\tau} d_{i, k} \geq d_{k}$, for any $1 \leq k \leq n-1$. This property will later admit the construction of a feasible schedule in which the computing demands of $\mathcal{I}$ are met. (3) Given the flow of Step 2, $\mathcal{A}_{2}$ packs fractional flows on the lower paths of the components $C_{1}, \ldots, C_{\tau}$. Using the integral flow obtained in Step 3, $\mathcal{A}_{2}$ constructs a feasible schedule for $\mathcal{I}$ whose energy consumption is upper bounded by the cost of that flow. Once $f^{*}$ has been scaled in Step 1, the subsequent flow modifications of Steps 2 and 3 never increase cost. Thus, the energy consumed by the schedule is at most $\tau \cos t\left(f^{*}\right)$.

LEMMA 4.4. For $k=1, \ldots, n-1$, we have $\sum_{i=1}^{\tau} d_{i, k} \geq d_{k}$.

Proof. Fix any $k, 1 \leq k \leq n-1$. For any $i$ with $1 \leq i \leq \tau$, let $f^{*}\left(u_{i, k}, u_{i, k+1}\right)=m_{i, k}+\delta_{i, k}$, where $m_{i, k} \in \mathbb{N}_{0}$ and $0 \leq \delta_{i, k}<1$. Set $\delta_{k}=\sum_{i=1}^{\tau} \delta_{i, k}$. We first argue that there exist at least $\left\lfloor\delta_{k}\right\rfloor$ values $i \in\{1, \ldots, \tau\}$ such that $\delta_{i, k} \geq 1 / \tau$. If $\left\lfloor\delta_{k}\right\rfloor=0$, there is nothing to show. So suppose that $\left\lfloor\delta_{k}\right\rfloor \geq 1$. If there were less than $\left\lfloor\delta_{k}\right\rfloor$ values $i$ such that $\delta_{i, k} \geq 1 / \tau$, then there would be at most $\left\lfloor\delta_{k}\right\rfloor-1$ values $i$ with $1 / \tau \leq \delta_{i, k}<1$. For the remaining values $i$, there holds $\delta_{i, k}<1 / \tau$. Hence, $\sum_{i=1}^{\tau} \delta_{i, k}<\left\lfloor\delta_{k}\right\rfloor-1+\left(\tau-\left\lfloor\delta_{k}\right\rfloor+1\right) / \tau=\left\lfloor\delta_{k}\right\rfloor+\left(1-\left\lfloor\delta_{k}\right\rfloor\right) / \tau \leq \delta_{k}$, which is a contradiction to the definition of $\delta_{k}$. The last inequality uses the fact that $\left\lfloor\delta_{k}\right\rfloor \geq 1$.

For any $i$ such that $\delta_{i, k} \geq 1 / \tau$, there holds $\left\lfloor\tau f^{*}\left(u_{i, k}, u_{i, k+1}\right)\right\rfloor \geq\left\lfloor\tau\left(m_{i, k}+1 / \tau\right)\right\rfloor \geq m_{i, k}+1$. Moreover, $m_{i, k}+1 \leq m_{i}$ because $\delta_{i, k}>0$, and thus $m_{i, k}<f^{*}\left(u_{i, k}, u_{i, k+1}\right)<m_{i}$. For any $i$ such that $\delta_{i, k}<1 / \tau$, there holds $\left\lfloor\tau f^{*}\left(u_{i, k}, u_{i, k+1}\right)\right\rfloor \geq m_{i, k}$. In addition, $m_{i, k} \leq m_{i}$. As shown in the last paragraph, there exist at least $\left\lfloor\delta_{k}\right\rfloor$ values $i \in\{1, \ldots, \tau\}$ such that $\delta_{i, k} \geq 1 / \tau$. Considering the definition of $d_{i, k}$, see (2), we obtain $\sum_{i=1}^{\tau} d_{i, k} \geq \sum_{i=1}^{\tau} m_{i, k}+\left\lfloor\delta_{k}\right\rfloor=\left\lfloor\sum_{i=1}^{\tau} m_{i, k}+\delta_{k}\right\rfloor=\left\lfloor\sum_{i=1}^{\tau}\left(m_{i, k}+\right.\right.$ $\left.\left.\delta_{i, k}\right)\right\rfloor=\left\lfloor\sum_{i=1}^{\tau} f^{*}\left(u_{i, k}, u_{i, k+1}\right)\right\rfloor \geq d_{k}$. The last inequality follows from Lemma 4.2 and the fact that $d_{k}$ is an integer value.

Given Lemma 4.4, a natural idea for finding an integral solution is to use a flow computation: Determine a single-commodity minimum-cost flow that ships $m_{i}$ flow units through component $C_{i}$ and, importantly, exactly $d_{i, k}$ units along edge $\left(u_{i, k}, u_{i, k+1}\right)$, where $1 \leq i \leq \tau$ and $1 \leq k \leq n-1$. However, one has to prove that the cost of such a flow is upper bounded by $\tau \cos t\left(f^{*}\right)$. Such a proof involves arguments and flow modifications contained in Steps 1 and 2 of $\mathcal{A}_{2}$. Therefore, we describe them explicitly as algorithmic steps. Step 3 could indeed be replaced by a min-cost flow computation. However, we instead devise a faster $O\left(n^{2} \sum_{i=1}^{\tau} \sigma_{i}\right)$ time routine for constructing an integral flow along the lower paths of the components.

In the following, when describing flow modifications, we will always focus on one particular network component. All flow updates will be performed independently for the components. Hence, in the corresponding exposition, we consider an arbitrary but fixed component $C=C_{i}, 1 \leq i \leq$ $\tau$. This allows us to simplify notation and omit the index $i$. On the upper path, the vertices are $u_{1}, \ldots, u_{n}$. Component $C$ has $\sigma=\sigma_{i}$ lower paths. On the lower path $j, 1 \leq j \leq \sigma$, the vertices are $l_{j, k}$ and $l_{j, k}^{a}$, for $k=1, \ldots, n-1$, followed by the final vertex $l_{j, n}$. Let $m_{c}=m_{i}$ be the number of servers in class $\mathcal{S}_{i}$ represented by $C=C_{i}$.

Nested structure of flows. We show that in each network component $C$ flow $f^{*}$ has a crucial property, i.e., it exhibits a nested structure. Let $P_{j}\left(k, k^{\prime}\right)$ be the path from $u_{k}$ to $u_{k^{\prime}}$ along lower path $j$, where $1 \leq j \leq \sigma$ and $1<k<k^{\prime}<n$. More specifically, the path consists of $\left(u_{k}, l_{j, k}\right)$, followed by the path from $l_{j, k}$ to $l_{j, k^{\prime}}$ on lower path $j$, followed by $\left(l_{j, k^{\prime}}, u_{k^{\prime}}\right)$. For $k=1$ and $1<k^{\prime}<n$, we define $P_{\sigma}\left(1, k^{\prime}\right)$ as the path consisting of the edges from $l_{\sigma, 1}$ to $l_{\sigma, k^{\prime}}$ on lower path $\sigma$, followed by the edge $\left(l_{\sigma, k^{\prime}}, u_{k^{\prime}}\right)$. For $k^{\prime}=n$ and $1<k<n$, path $P_{\sigma}(k, n)$ consists of edge $\left(u_{k}, l_{\sigma, k}\right)$, followed by 


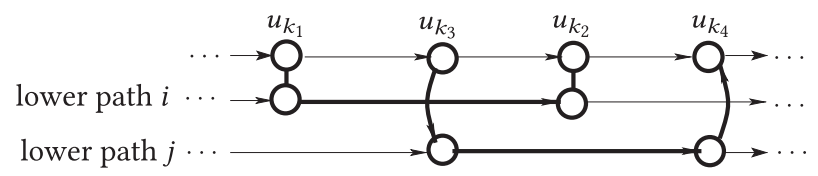

(a)

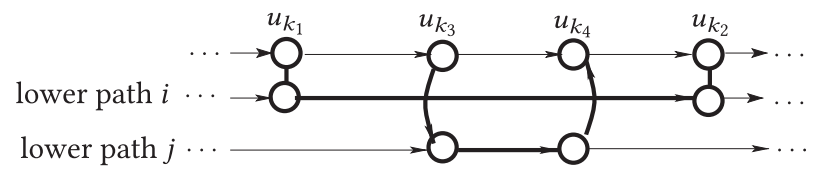

(b)

Fig. 9. Paths that are not nested. Only the relevant vertices and edges are shown.

the edges from $l_{\sigma, k}$ to $l_{\sigma, n}$ on lower path $\sigma$. Finally, $P\left(k, k^{\prime}\right)$ is the path connecting $u_{k}$ and $u_{k^{\prime}}$ on the upper path of the component, for any $1 \leq k<k^{\prime} \leq n$. In the sequel, unless otherwise stated, flow always refers to commodity 1 . Consider any path $P$. We say that $P$ routes flow if, for any edge of $P$, the flow is strictly positive.

The following property of a flow will be important. A flow $f$ in component $C$ is nested if it satisfies the following condition. Let $P_{i}\left(k_{1}, k_{2}\right)$ and $P_{j}\left(k_{3}, k_{4}\right)$ be two paths such that both route flow and $i<j$. Then one of the relations (a-c) holds: (a) $k_{2}<k_{3}$, (b) $k_{4}<k_{1}$, or (c) $k_{3} \leq k_{1}<k_{2} \leq k_{4}$. Intuitively, the endpoints of the two paths do not alternate. Both endpoints of $P_{i}\left(k_{1}, k_{2}\right)$ occur either before, after, or in between those of $P_{j}\left(k_{3}, k_{4}\right)$. Figure 9 show two forbidden structures of paths that are not nested.

\section{Lemma 4.5. In each component $C$, flow $f^{*}$ is nested.}

Proof. Let $P_{i}\left(k_{1}, k_{2}\right)$ and $P_{j}\left(k_{3}, k_{4}\right)$, with $i<j$, be two paths that route flow. We assume that none of the relations $(\mathrm{a}-\mathrm{c})$ holds and derive a contradiction to the optimality of $f^{*}$. Determine a $\delta>0$ such that $\delta$ units of flow a routed on all edges of $P_{i}\left(k_{1}, k_{2}\right)$ and $P_{j}\left(k_{3}, k_{4}\right)$.

First assume that $k_{2}=k_{3}$. Considering that $i<j \leq \sigma$, there holds $1<k_{1}$. We modify $f^{*}$ in the following way. On $P_{i}\left(k_{1}, k_{2}\right)$, we delete $\delta$ units of flow and instead route this flow on $P_{j}\left(k_{1}, k_{2}\right)$ using lower path $j$. We then delete $\delta$ units of flow entering/leaving $u_{k_{2}}$. More specifically, we delete $\delta$ units of flow on $\left(l_{j, k_{2}}, u_{k_{2}}\right)$ and on $\left(u_{k_{2}}, l_{j, k_{2}}\right)$. The flow conservation law is observed at vertices $l_{j, k_{2}}$ and $u_{k_{2}}$. The flow of commodity 2 can be adapted easily: For any $k$ with $k_{1} \leq k<k_{2}$, up to $\delta$ units of flow that cannot be shipped anymore from $a_{k}$ to $b_{k}$ using lower path $j$ can now be routed via lower path $i$. The new flow has a strictly smaller cost. The cost saving is $\delta\left(r_{i}-r_{j}\right)\left(t_{k_{2}}-t_{k_{1}}\right)+\delta \Delta_{i}>0$. The last inequality holds because $r_{i}>r_{j}$. Here, $r_{i}$ and $r_{j}$ are the cost coefficients on lower path $i$ and $j$. More precisely, for any $1 \leq k \leq n-1$, the cost of edges $\left(l_{i, k}, l_{i, k}^{a}\right)$ and $\left(l_{j, k}, l_{j, k}^{a}\right)$ is $r_{i}\left(t_{k+1}-t_{k}\right)$ and $r_{j}\left(t_{k+1}-t_{k}\right)$, respectively. Moreover, $\Delta_{i}$ is the cost of an edge connecting lower path $i$ to the upper path. In the same way, we can handle the case $k_{4}=k_{1}$. Then $k_{2}<n$ holds.

Next, assume that $k_{1}<k_{3}<k_{2} \leq k_{4}$. Again, $1<k_{1}$. We remove $\delta$ units of flow from both $P_{i}\left(k_{1}, k_{2}\right)$ and $P_{j}\left(k_{3}, k_{4}\right)$. Instead, we route $\delta$ units of flow on $P_{i}\left(k_{3}, k_{2}\right)$ and $P_{j}\left(k_{1}, k_{4}\right)$. Both of these paths exist. If $k_{4}<n$, this is obvious. If $k_{4}=n$, then $k_{2}<k_{4}$ because $i<j \leq \sigma$. Path $P_{i}\left(k_{3}, k_{2}\right)$ exists because $1<k_{3}<k_{2}<n$, and $P_{j}\left(k_{1}, k_{4}\right)$ exists because $1<k_{1}$. Again, we can easily update commodity 2. The flow modifications save a cost of $\delta\left(r_{i}-r_{j}\right)\left(t_{k_{3}}-t_{k_{1}}\right)>0$. Analogously, we can argue if $k_{3} \leq k_{1}<k_{4}<k_{2}$.

It remains to analyze the case $k_{1}<k_{3}<k_{4}<k_{2}$. There holds $1<k_{1}$ and $k_{2}<n$. We remove $\delta$ units of flow from $P_{i}\left(k_{1}, k_{2}\right)$ and $P_{j}\left(k_{3}, k_{4}\right)$. Instead, we ship $\delta$ units of flow on $P_{j}\left(k_{1}, k_{2}\right)$ 
and $P_{i}\left(k_{3}, k_{4}\right)$. Flow of commodity 2 is modified accordingly. The new flow saves a cost of $\delta\left(r_{i}-r_{j}\right)\left(t_{k_{3}}-t_{k_{1}}+t_{k_{2}}-t_{k_{4}}\right)>0$.

Loop freeness. Given $f^{*}, \mathcal{A}_{2}$ slightly modifies it so that it becomes loop free in each $C$. A flow is loop free in $C$ if there exists no vertex $u_{k}$ such that edges $\left(l_{i, k}, u_{k}\right)$ and $\left(u_{k}, l_{j, k}\right)$ both route flow, where $1 \leq i, j \leq \sigma$. Suppose that there exists such a vertex $u_{k}$. Considering that $f^{*}$ is nested, $i=j$ must hold. Otherwise, the would exist paths $P_{i}\left(k_{1}, k\right)$ and $P_{j}\left(k, k_{2}\right)$ with $k_{1}<k<k_{2}$ that route flow. As $i=j, \mathcal{A}_{2}$ can simply remove $\min \left\{f^{*}\left(l_{i, k}, u_{k}\right), f^{*}\left(u_{k}, l_{i, k}\right)\right\}$ units of flow from both $\left(l_{i, k}, u_{k}\right)$ and $\left(u_{k}, l_{i, k}\right)$. The flow conservation law is observed at $l_{i, k}$ and $u_{k}$. The cost of the flow can only decrease. By performing these updates, one obtains a loop-free flow $f^{*}$ that is nested.

\subsection{Constructing an Integral Flow}

We describe the three main modification steps.

Step 1: Flow scaling. Let $f^{*}$ be the minimum-cost, loop-free flow. Algorithm $\mathcal{A}_{2}$ multiplies $f^{*}$ by a factor of $\tau$ on all edges of the network. At the same time, it multiplies all edge capacities and supplies/demands by $\tau$. Then it deletes the flow of commodity 2 and the supplies/demands at $a_{k}$ and $b_{k}, 1 \leq k \leq n-1$. The resulting flow $f^{1}$ of commodity 1 is feasible. Additionally, in each component, it is nested and loop free. There holds $\cos t\left(f^{1}\right) \leq \tau \cos t\left(f^{*}\right)$.

In Steps 2 and 3, flow $f^{1}$ is modified. As indicated earlier, the modification are executed independently for the components. Therefore, in the description of Steps 2 and 3, we concentrate on one component $C$ that ships $\tau m_{c}$ units of flow. The flow modifications never increase the cost. At all times, the flow remains nested and loop free.

Step 2: Rounding flow on the upper path. Given $f^{1}, \mathcal{A}_{2}$ rounds it so the flow becomes integral on the upper path of $C$. On $\left(u_{k}, u_{k+1}\right)$, the flow will be $\min \left\{m_{c},\left\lfloor\tau f^{*}\left(u_{k}, u_{k+1}\right)\right\rfloor\right\}$. Recall that $m_{c}$ is the number of servers in the class represented by $C$. We first describe how to reduce $f^{1}$ so that on any edge $\left(u_{k}, u_{k+1}\right)$ the flow is $\left\lfloor\tau f^{*}\left(u_{k}, u_{k+1}\right)\right\rfloor$. $\mathcal{A}_{2}$ makes four passes over $C$. First, in Step 2.1, it rounds valleys with a nonintegral amount of flow. Then, in Steps 2.2 and 2.3, it modifies flow on edge sequences with increasing and decreasing flow, respectively. Finally, in Step 2.4, it takes care of flow peaks. Given this flow, we then describe how to further reduce it so that the flow on any edge of the upper path does not exceed $m_{c}$. At any time, for a current flow $f$, we say that the flow increases at $u_{k}$ if $k=1$ and $f\left(u_{1}, u_{2}\right)>0$ or if $1<k<n$ and $f\left(u_{k-1}, u_{k}\right)<f\left(u_{k}, u_{k+1}\right)$. Similarly, the flow decreases at $u_{k}$ if $1<k<n$ and $f\left(u_{k-1}, u_{k}\right)>f\left(u_{k}, u_{k+1}\right)$ of if $k=n$ and $f\left(u_{n-1}, u_{n}\right)>0$. Initially in Step 2, let $f=f^{1}$.

Step 2.1: Valleys. A valley is a path $P\left(k, k^{\prime}\right), 1<k<k^{\prime}<n$, on the upper path of $C$ such that the flow decreases at $u_{k}$, increases at $u_{k^{\prime}}$ and is constant for all edges of $P\left(k, k^{\prime}\right)$. Formally, the last condition indicates that $f(e)=f\left(u_{k}, u_{k+1}\right)$, for all edges $e$ of $P\left(k, k^{\prime}\right) . \mathcal{A}_{2}$ scans $C$. Whenever it encounters a valley $P\left(k, k^{\prime}\right)$ with a nonintegral amount of flow, it invokes the following procedure that reduces the flow to $\left\lfloor f\left(u_{k}, u_{k+1}\right)\right\rfloor$.

Flow update procedure. For the given valley $P\left(k, k^{\prime}\right)$, the flow decreases at $u_{k}$. The procedure determines the smallest integer $j$ such that flow is routed from $u_{k}$ to $l_{j, k}$ and shipped on lower path $j$.

Claim 1. $P_{j}\left(k, k^{\prime}\right)$ routes flow.

Proof. Suppose that $\delta_{j}$ units of flow are routed from $u_{k}$ to $l_{j, k}$. The flow along $P\left(k, k^{\prime}\right)$ is constant. Considering that the flow is loop free in $C$, no flow is routed from a vertex $l_{j, k^{\prime \prime}}$ to $u_{k^{\prime \prime}}$, where $k<k^{\prime \prime}<k^{\prime}$. Thus, all edges between $l_{j, k}$ and $l_{j, k^{\prime}}$ on path $P_{j}\left(k, k^{\prime}\right)$ carry $\delta_{j}$ units of flow. This implies that $\delta_{j}$ units of flow reach $l_{j, k^{\prime}}$ via lower path $j$. If none of these flow units are shipped to 
$u_{k^{\prime}}$, then the flow of $C$ would not be nested: A path $P_{j}\left(k, k_{3}\right)$ with $k_{3}>k^{\prime}$ would route flow. Furthermore, a path $P_{i}\left(k_{4}, k^{\prime}\right)$ with $i \neq j$ and $k_{4} \leq k$ would carry flow into $u_{k^{\prime}}$. This holds true because the flow increases at $u_{k^{\prime}}$ and the flow is loop free in $C$. If $i<j$, then by the choice of $j$ there holds $k_{4}<k$ and the flow of $C$ is not nested. If $i>j$, then the flow is not nested either, even if $k_{4}=k$.

Hence, as $P_{j}\left(k, k^{\prime}\right)$ routes flow, in the unscaled minimum-cost flow $f^{*}$ path $P_{j}\left(k, k^{\prime}\right)$ also ships flow. Routing the flow on the upper path would have been a feasible option as well. This implies that the total edge cost of $P_{j}\left(k, k^{\prime}\right)$ is upper bounded by that of $P\left(k, k^{\prime}\right)$. The procedure updates the flow as follows. It remove $\delta=f\left(u_{k}, u_{k+1}\right)-\left\lfloor f\left(u_{k}, u_{k+1}\right)\right\rfloor$ units of flow from $P\left(k, k^{\prime}\right)$ and instead routes them along $P_{j}\left(k, k^{\prime}\right)$. This does not increase the cost. The resulting flow in $C$ remains nested. Modifying all valleys takes $O(n \sigma)$ time.

Step 2.2: Flow increases. In a second pass over $C$ algorithm $\mathcal{A}_{2}$ identifies vertices $u_{k}$ at which the flow increases. If $f\left(u_{k}, u_{k+1}\right)$ is not integral, it is reduced to $\left\lfloor f\left(u_{k}, u_{k+1}\right)\right\rfloor$. Starting at $u_{1}$ or at a vertex representing the end of a valley, $\mathcal{A}_{2}$ performs a sequence of vertex inspections and possible flow updates. The sequence ends at a vertex at which the flow decreases. The algorithm then searches for the end of the next valley and continues.

Formally, let $u_{k^{\prime}}$ be a vertex such that $k^{\prime}=1$ or $u_{k^{\prime}}$ is the last vertex of a valley. When located at $u_{k^{\prime}}, \mathcal{A}_{2}$ determines the smallest $k^{\prime \prime}$ with $k^{\prime \prime}>k^{\prime}$ such that the flow decreases at $u_{k^{\prime \prime}}$. The algorithm inspects the vertices $u_{k}, k^{\prime} \leq k<k^{\prime \prime}-1$, in order of increasing index. If $f\left(u_{k}, u_{k+1}\right)$ is not integral, the procedure described in the next paragraph is invoked, which reduces the flow to $\left\lfloor f\left(u_{k}, u_{k+1}\right)\right\rfloor$. When the procedure is executed at $u_{k}$, we have $u_{k}=u_{1}$ or the flow $f\left(u_{k-1}, u_{k}\right)$ on the preceding edge is integral. The latter condition holds true because if $u_{k}=u_{k^{\prime}}$ is the last vertex of a valley, then the flow along the incoming edge has been made integral in Step 2.1. $\mathcal{A}_{2}$ considers vertices in order of increasing index, starting at $u_{k^{\prime}}$. When $u_{k}, k^{\prime}<k<k^{\prime \prime}-1$, is inspected, the flow on the edges between $u_{k^{\prime}}$ and $u_{k}$ is already integral. We note that if $f\left(u_{k}, u_{k+1}\right)$ is reduced by a call of the procedure, then $u_{k+1}$ becomes a vertex where the flow increases, provided that this has not been the case before. We also remark that flow on $\left(u_{k^{\prime \prime}-1}, u_{k^{\prime \prime}}\right)$ is not modified. If $f\left(u_{k^{\prime \prime}-1}, u_{k^{\prime \prime}}\right)$ is not integral, then $\left(u_{k^{\prime \prime}-1}, u_{k^{\prime \prime}}\right)$ is a peak when $\mathcal{A}_{2}$ reaches $u_{k^{\prime \prime}-1}$, i.e., the flow increases at $u_{k^{\prime \prime}-1}$ and decreases at $u_{k^{\prime \prime}}$. Peaks will be handled in Step 2.4. When located at $u_{k^{\prime \prime}}, \mathcal{A}_{2}$ moves to the end of the next valley, if it exists.

Flow update procedure. The procedure that updates flow at a vertex $u_{k}$ where the flow increases and $f\left(u_{k}, u_{k+1}\right)$ is not integral. Let again $\delta=f\left(u_{k}, u_{k+1}\right)-\left\lfloor f\left(u_{k}, u_{k+1}\right)\right\rfloor$. Either $k=1$ or the flow on $\left(u_{k-1}, u_{k}\right)$ is integral. Hence, at least $\delta$ units of flow are shipped from lower paths into $u_{k}$. While $\delta>0$, the procedure executes the following steps. Let $j$ be the largest integer such that the flow from $l_{j, k}$ to $u_{k}$ is positive. Figure 10(a) depicts the general flow configuration. Let $\delta_{j}=f\left(l_{j, k}, u_{k}\right)$ and $\delta^{\prime}=\min \left\{\delta, \delta_{j}\right\}$. The procedure reduces flow on $\left(l_{j, k}, u_{k}\right)$ and $\left(u_{k}, u_{k+1}\right)$ by $\delta$ units. Instead, it ships $\delta$ units of flow from $l_{j, k}$ to $u_{k+1}$ along lower path $j$, i.e., via $l_{j, k}^{a}$ and $l_{j, k+1}$. Then $\delta$ is reduced by $\delta^{\prime}$. The flow update decreases the cost of the flow by $\left(r_{0}-r_{j}\right)\left(t_{k+1}-t_{k}\right)>0$. Here, $r_{0}$ and $r_{j}$ are the cost coefficients along the upper path and lower path $j$, respectively. More precisely, edge $\left(u_{k}, u_{k+1}\right)$ has a cost of $r_{0}\left(t_{k+1}-t_{k}\right)$ and $\left(l_{j, k}, l_{j, k}^{a}\right)$ has a cost of $r_{j}\left(t_{k+1}-t_{k}\right)$. The modified flow remains nested: By the choice of $j$, there exists no path $P_{j^{\prime}}\left(k_{1}, k\right)$ with $j^{\prime}>j$ and $k_{1}<k$ that routes flow. Moreover, since the flow does not decrease at $u_{k+1}$ and is loop free, there is no path $P_{j^{\prime}}\left(k+1, k_{2}\right), 1 \leq j^{\prime} \leq \sigma$ and $k+1<k_{2}$, that routes flow. The running time of one execution of the procedure is $O(\sigma)$. The running time of the entire pass over $C$ is $O(n \sigma)$.

Step 2.3: Flow decreases. The flow modifications are symmetric to those described in Step 2.2. Algorithm $\mathcal{A}_{2}$ makes another pass over $C$, this time from right to left starting at $u_{n}$. It searches for vertices $u_{k}$ at which the flow decreases. If the flow $f\left(u_{k-1}, u_{k}\right)$ on the incoming edge is not 


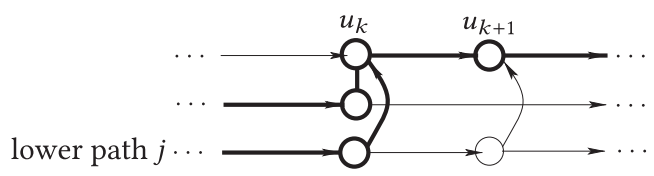

(a)

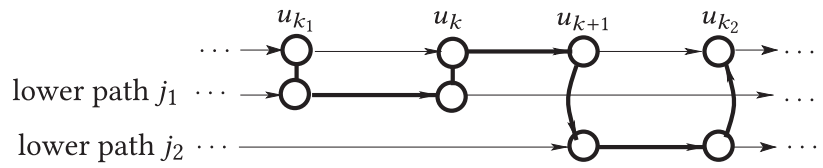

(b)

Fig. 10. Flow configurations. (a) A flow increase. (b) A peak.

integral, then it is reduced to $\left\lfloor f\left(u_{k-1}, u_{k}\right)\right\rfloor$. Let $u_{k^{\prime \prime}}$ be a vertex such that $u_{k^{\prime \prime}}=n$ or $u_{k^{\prime \prime}}$ is the first vertex of a valley. When located at $u_{k^{\prime \prime}}, \mathcal{A}_{2}$ determines the largest $k^{\prime}$ with $k^{\prime}<k^{\prime \prime}$ such that $u_{k^{\prime}}$ is a vertex where the flow increases. Then it considers the vertices $u_{k}, k^{\prime}+1<k \leq k^{\prime \prime}$, in order of decreasing index. If $f\left(u_{k-1}, u_{k}\right)$ is not integral, $\mathcal{A}_{2}$ invokes the procedure described in the next paragraph. When the procedure is executed for $u_{k}$, we have $k=n$ or the flow $f\left(u_{k}, u_{k+1}\right)$ on the outgoing edge is integral. The last condition holds because vertices are inspected in order of decreasing index starting at $u_{k^{\prime \prime}}$ such that $k^{\prime \prime}=n$ or $u_{k^{\prime \prime}}$ is the first vertex of a valley, for which the flow along the outgoing edge is integral. When $\mathcal{A}_{2}$ reaches $u_{k}$, the flow on the edges between $u_{k}$ and $u_{k^{\prime \prime}}$ is integral. Moreover, when the procedure has been executed at $u_{k}$, the flow decreases at $u_{k-1}$. When reaching $u_{k^{\prime}+1}, \mathcal{A}_{2}$ does not adjust flow on $\left(u_{k^{\prime}}, u_{k^{\prime}+1}\right)$, as this edge is a peak. Instead, it searches for the first vertex of the preceding valley and continues.

Flow update procedure. Given a vertex $u_{k}$ where the flow decreases, the procedure works as follows. Let $\delta=f\left(u_{k-1}, u_{k}\right)-\left\lfloor f\left(u_{k-1}, u_{k}\right)\right\rfloor$. While $\delta>0$, the procedure determines the largest $j$ such that flow is routed from $u_{k}$ to $l_{j, k}$. Let $\delta_{j}=f\left(u_{k}, l_{j, k}\right)$ and $\delta^{\prime}=\min \left\{\delta, \delta_{j}\right\}$. The procedure reduces the flow on $\left(u_{k-1}, u_{k}\right)$ and $\left(u_{k}, l_{j, k}\right)$ by $\delta$ units. Instead, the flow is shipped from $u_{k-1}$ to $l_{j, k}$ via $l_{j, k-1}$ and $l_{j, k-1}^{a}$. Then $\delta$ is reduced by $\delta^{\prime}$. The modified flow has a strictly smaller cost; the savings is $\left(r_{0}-r_{j}\right)\left(t_{k}-t_{k-1}\right)$. It is still nested because there is no path $P_{j^{\prime}}\left(k, k_{1}\right), j^{\prime}>j$, that routes flow. Moreover, there is no path $P_{j^{\prime}}\left(k_{2}, k-1\right)$, with $k_{2}<k-1$, that routes flow because the flow on the upper path does not increase at $u_{k-1}$. The entire scan over $C$ takes $O(n \sigma)$ time.

Step 2.4: Peaks. A peak is an edge $\left(u_{k}, u_{k+1}\right)$ such that the flow increases at $u_{k}$ and decreases at $u_{k+1}$. After $\mathcal{A}_{2}$ has executed Steps 2.1 through 2.3, the only edges on the upper path with a nonintegral amount of flow are peaks: Edges considered but not handled in Step 2.2 end in vertices $u_{k^{\prime \prime}}$ at which the flow decreases. Such a vertex $u_{k^{\prime \prime}}$ is followed by vertices at which the flow does not increase. The vertex sequence ends at the beginning of a valley or at $u_{n}$. The edges of the corresponding path are handled in Step 2.3. If $f\left(u_{k^{\prime \prime}-1}, u_{k^{\prime \prime}}\right)$ is not integral, then the flow at $u_{k^{\prime \prime}-1}$ increases because the flow along $f\left(u_{k^{\prime \prime}-2}, u_{k^{\prime \prime}-1}\right)$ is integral.

Algorithm $\mathcal{A}_{2}$ traverses $C$. For each peak $\left(u_{k}, u_{k+1}\right)$ with a nonintegral amount of flow, it invokes the following routine.

Flow update procedure. As always, $\delta=f\left(u_{k}, u_{k+1}\right)-\left\lfloor f\left(u_{k}, u_{k+1}\right)\right\rfloor$. Let $j_{1}$ be the largest integer such that $f\left(l_{j_{1}, k}, u_{k}\right)>0$, i.e., flow is routed from lower path $j_{1}$ to $u_{k}$. Furthermore, let $j_{2}$ be the largest integer such that $f\left(u_{k+1}, l_{j_{2}, k+1}\right)>0$. There are two basic cases. We describe the flow update and then show that the new flow remains nested.

If $j_{1}=j_{2}$, then let $\delta_{1}=f\left(l_{j_{1}, k}, u_{k}\right)$ and $\delta_{2}=f\left(u_{k+1}, l_{j_{2}, k+1}\right)$. Furthermore, let $\delta^{\prime}=\min \left\{\delta, \delta_{1}, \delta_{2}\right\}$. The procedure removes $\delta^{\prime}$ units of flow from the path connecting $l_{j_{1}, k}$ and $l_{j_{1}, k+1}$ along the upper 
path. Specifically, it removes $\delta^{\prime}$ units of flow from the edges $\left(l_{j_{1}, k}, u_{k}\right),\left(u_{k}, u_{k+1}\right)$ and $\left(u_{k+1}, l_{j_{1}, k+1}\right)$. Instead, it sends $\delta^{\prime}$ units of flow from $l_{j_{1}, k}$ to $l_{j_{1}, k+1}$ via $l_{j_{1}, k}^{a}$ on lower path $j_{1}$. The reduction in the cost of the flow is $\delta\left(r_{0}-r_{j_{1}}\right)\left(t_{k+1}-t_{k}\right)+\delta \Delta_{j_{1}}>0$. Here, $\Delta_{j}$ is the cost of $\left(l_{j, k}, u_{k}\right)$, for any $1 \leq j \leq \sigma$ and $1 \leq k<n-1$.

If $j_{1} \neq j_{2}$, then let $k_{1}$ be the smallest integer such that $P_{j_{1}}\left(k_{1}, k\right)$ routes flow. Let $\delta_{1}$ be the largest value such that every edge of $P_{j_{1}}\left(k_{1}, k\right)$ routes at least $\delta_{1}$ units of flow. Similarly, let $k_{2}$ be the largest integer such that $P_{j_{2}}\left(k+1, k_{2}\right)$ routes flow. Let $\delta_{2}$ be the largest value such that every edge of $P_{j_{2}}\left(k+1, k_{2}\right)$ routes at least $\delta_{2}$ units of flow. Figure 10(b) shows the case that $j_{1}<j_{2}$. Let $\delta^{\prime}=\min \left\{\delta, \delta_{1}, \delta_{2}\right\}$. The procedure removes $\delta^{\prime}$ units of flow from $P_{j_{1}}\left(k_{1}, k\right)$, edge $\left(u_{k}, u_{k+1}\right)$, and $P_{j_{2}}\left(k+1, k_{2}\right)$. If $j_{1}<j_{2}$, it instead sends these $\delta^{\prime}$ units on path $P_{j_{2}}\left(k_{1}, k_{2}\right)$. If $j_{1}>j_{2}$, it routes the $\delta^{\prime}$ units along $P_{j_{1}}\left(k_{1}, k_{2}\right)$. Thus, in any case, the deeper low-power state is used. Note that the flow conservation law is maintained, particularly at vertices $u_{k_{1}}$ and $u_{k_{2}}$. In case $j_{1}<j_{2}$, the reduction in the cost of the flow is $\delta^{\prime}\left(\left(r_{j_{1}}-r_{j_{2}}\right)\left(t_{k}-t_{k_{1}}\right)+\Delta_{j_{1}}+\left(r_{0}-r_{j_{2}}\right)\left(t_{k+1}-t_{k}\right)\right)$. If $j_{1}>j_{2}$, the reduction in the cost of the flow is $\delta^{\prime}\left(\left(r_{0}-r_{j_{1}}\right)\left(t_{k+1}-t_{k}\right)+\left(r_{j_{2}}-r_{j_{1}}\right)\left(t_{k_{2}}-t_{k+1}\right)+\Delta_{j_{1}}\right)$.

In any case, $\delta$ is reduced by $\delta^{\prime}$. The next claim ensures that the new flow is nested. One call of the procedure takes $O\left(n^{2} \sigma\right)$ time such that the rounding of all peaks can be accomplished in $O\left(n^{3} \sigma\right)$ time.

\section{Claim 2. The modified flow is nested.}

Proof. Consider the flow before modification. First, assume that $j_{1}=j_{2}$. By the choice of $j_{1}$ and $j_{2}$, there exist no paths $P_{j}\left(k_{1}, k\right)$ or $P_{j}\left(k+1, k_{2}\right)$, where $j>j_{1}=j_{2}$ and $k_{1}<k<k+1<k_{2}$, that route flow. Thus, the flow shipment along lower path $j_{1}$ preserves the nested structure of the flow.

Next, assume $j_{1}<j_{2}$. We show that there exists no path $P_{j}\left(k_{3}, k_{4}\right)$ with $j_{1} \leq j<j_{2}$ and $k_{3}<$ $k_{1}<k_{4}$ that routes flow. This ensures that the routing of flow on $P_{j_{2}}\left(k_{1}, k_{2}\right)$ preserves the nested structure of the flow. First, consider $j=j_{1}$. If there were a path $P_{j_{1}}\left(k_{3}, k_{4}\right)$ with $k_{3}<k_{1}<k_{4}$ that routes flow, then $P_{j_{1}}\left(k_{3}, k\right)$ would also route flow, which contradicts the choice of $k_{1}$. Next, consider any $j$ with $j_{1}<j<j_{2}$. We argue that edge $\left(l_{j, k_{1}}, l_{j, k_{1}}^{a}\right)$ cannot route flow. Flow along this edge cannot be shipped to vertex $u_{k^{\prime}}$ with $k_{1}<k^{\prime}<k$ because this would imply the existence of a path $P_{j}\left(k_{3}, k^{\prime}\right)$ with $k_{3} \leq k_{1}$ that routes flow. This in turn would contradict the fact that the flow is nested. By the choice of $j_{1}$, the flow cannot be routed to $u_{k}$. Hence, the flow must reach $l_{j, k+1}$. If $k+1=n$, then the flow cannot reach $l_{\sigma, n}$ at the end of the component $C$. Thus, the flow in $C$ would not be feasible. Assume that $k+1<n$. The flow arriving at $l_{j, k+1}$ is not sent to $u_{k+1}$, because the flow is loop free. Hence, there must exist a path $P_{j}\left(k_{3}, k_{4}\right)$, where $k_{3} \leq k_{1}<k+1<k_{4}$, that routes flow. Considering that $P_{j_{2}}\left(k+1, k_{2}\right)$ routes flow, the flow in $C$ would not be nested. It follows that no edge $\left(l_{j, k_{1}}, l_{j, k_{1}}^{a}\right), j_{1}<j<j_{2}$, routes flow. Consequently, there exists no path $P_{j}\left(k_{3}, k_{4}\right)$ with $j_{1}<j<j_{2}$ and $k_{3}<k_{1}<k_{4}$ that routes flow.

The analysis of $j_{1}>j_{2}$ is similar. As in the previous paragraph, one can show that there exists no path $P_{j}\left(k_{3}, k_{4}\right)$ with $j_{1}>j \geq j_{2}$ and $k_{3}<k_{2}<k_{4}$ that routes flow. Hence, the routing of flow on $P_{j_{1}}\left(k_{1}, k_{2}\right)$ observes the nested structure of the flow. For the case $j_{1}>j>j_{2}$, one can easily argue that there exists no edge $\left(l_{j, k_{2}-1}, l_{j, k_{2}-1}^{a}\right)$ that carries flow.

Step 2.5: Flow reduction to $\boldsymbol{m}_{\boldsymbol{c}}$. It remains to reduce the flow to $m_{c}$ on edges $\left(u_{k}, u_{k+1}\right)$ where the flow after Steps 2.1 through 2.4 is higher. This can be done using the procedures that handle flow increases and peaks. Again, $\mathcal{A}_{2}$ scans the vertices $u_{k}$ in order of increasing index $k$. Suppose that on the upper path the edges between $u_{1}$ and $u_{k}$ already ship a flow of at most $m_{c}$. Assume that $f\left(u_{k}, u_{k+1}\right)>m_{c}$. If the flow does not decrease at $u_{k+1}$, then $\mathcal{A}_{2}$ executes the procedure that handles flow increases. The value $\delta$ is set to $\delta=f\left(u_{k}, u_{k+1}\right)-m_{c}$. If the flow decreases at $u_{k+1}$, then $\mathcal{A}_{2}$ first invokes the procedure that handles peaks. If $k+1=n$, then the reduction value is $\delta=$ 
$f\left(u_{k}, u_{k+1}\right)-m_{c}$. Otherwise, it is $\delta=f\left(u_{k}, u_{k+1}\right)-\max \left\{m_{c}, f\left(u_{k+1}, u_{k+2}\right)\right\}$. If $f\left(u_{k+1}, u_{k+2}\right)>m_{c}$, then the algorithm then executes the procedure for handling flow increases with $\delta=f\left(u_{k}, u_{k+1}\right)-$ $m_{c}$, where $f\left(u_{k}, u_{k+1}\right)$ is the flow value after peak reduction. The running time is $O\left(n^{3} \sigma\right)$.

Step 3: Packing flow on the lower paths. Given the flow $f^{2}$ constructed in Step 2, $\mathcal{A}_{2}$ packs flow on the lower paths of the considered component $C$ so that the final flow becomes integral. During the modification, the flow on the upper path of $C$ does not change. Moreover, the cost of the flow will not increase.

Auxiliary edges. To separate flow that has already been made integral from the original one, we need auxiliary edges. For every edge $e$ in $C$, except for those on the upper path, we add an auxiliary edge $e^{\prime}$. More precisely, for every link $e=(v, w)$ not contained on the upper path, there is the original edge and a new auxiliary edge. Hence, during the flow packing operation, component $C$ is a multigraph.

Initially, the flow $f^{2}$ is routed on the upper path and the original edges of the lower paths. In a series of rounds, $\mathcal{A}_{2}$ removes flow from the original edges, packs it, and adds it to the auxiliary edges. On the auxiliary edges, the flow is always integral. The process ends when there is no flow on the original edges. Then the original edges are removed so that, for each edge, there is only one copy.

We observe that since $f^{2}$ is integral on the upper path and loop free, only integral amounts of flow enter/leave the upper path from/to the lower paths. At every vertex $u_{k}$ where the flow on the upper path increases, an integral amount of flow is routed into $u_{k}$ from lower paths. At every vertex $u_{k}$ where the flow decreases, an integral amount of flow is sent from $u_{k}$ to lower paths. This invariant will be maintained at all times during the flow transformation. Moreover, the flow will always be nested and loop free, considering the combined flow on the original and auxiliary edges. Last but not least, the flow will always be feasible. The flow conservation law is observed. For every edge, the total flow on the original and auxiliary copy is at most $\tau m_{c}$.

Matching pairs. The flow packing procedure uses the notion of a matching pair. A matching pair consists of two vertices $u_{k}$ and $u_{k^{\prime}}, 1<k<k^{\prime}<n$, with the following properties: (a) Flow is routed from $u_{k}$ to the lower paths on original edges $\left(u_{k}, l_{j, k}\right), 1 \leq j \leq \sigma$; (b) flow is routed into $u_{k^{\prime}}$ from lower paths on original edges $\left(l_{j, k^{\prime}}, u_{k^{\prime}}\right), 1 \leq j \leq \sigma$; and (c) there exists no vertex $u_{k^{\prime \prime}}$ with $k<k^{\prime \prime}<k^{\prime}$ that satisfies (a) or (b). While there exists a matching pair, $\mathcal{A}_{2}$ executes the following flow packing routine. Let $f$ denote the current flow. Unless stated otherwise, $f(e)$ always refers to the flow on the original copy of $e$.

Packing procedure. Let $u_{k}$ and $u_{k^{\prime}}$ be the given matching pair. Let $\delta_{k}$ be the total amount of flow routed from $u_{k}$ to lower paths on original edges $\left(u_{k}, l_{j, k}\right), 1 \leq j \leq \sigma$. Similarly, let $\delta_{k^{\prime}}$ be the total amount of flow shipped into $u_{k^{\prime}}$ from lower paths along original edges $\left(l_{j, k^{\prime}}, u_{k^{\prime}}\right), 1 \leq j \leq \sigma$. Both $\delta_{k}$ and $\delta_{k^{\prime}}$ are integral. If $\delta_{k} \leq \delta_{k^{\prime}}$, then let $J$ be the set of integers $j$ with $1 \leq j \leq \sigma$ such that $f\left(u_{k}, l_{j, k}\right)>0$. Define $\delta_{j}=f\left(u_{k}, l_{j, k}\right)$, for any $j \in J$. We have $\sum_{j \in J} \delta_{j}=\delta_{k}$. If $\delta_{k}>\delta_{k^{\prime}}$, then let $J$ be the set of integers $j$ with $1 \leq j \leq \sigma$ such that $f\left(l_{j, k^{\prime}}, u_{k^{\prime}}\right)>0$. Define $\delta_{j}=f\left(l_{j, k^{\prime}}, u_{k^{\prime}}\right)$, for any $j \in J$. There holds $\sum_{j \in J} \delta_{j}=\delta_{k^{\prime}}$. In any case, for $j \in J$, consider the path $P_{j}\left(k, k^{\prime}\right)$ from $u_{k}$ to $u_{k^{\prime}}$ along lower path $j$. We say that $P_{j}\left(k, k^{\prime}\right)$ routes $\delta$ units of flow if, for every original edge of $P_{j}\left(k, k^{\prime}\right)$, the amount of flow is at least $\delta$. Claim 3 states that $P_{j}\left(k, k^{\prime}\right)$ routes $\delta_{j}$ units of flow, for every $j \in J$.

The procedure for packing flow works as follows. It determines the integer $j^{\prime} \in J$ such that the total edge cost of $P_{j^{\prime}}\left(k, k^{\prime}\right)$ is minimal among $P_{j}\left(k, k^{\prime}\right)$ with $j \in J$. Then, for every $j \in J$, it removes $\delta_{j}$ units of flow from the original edges of $P_{j}\left(k, k^{\prime}\right)$. Finally, it routes $\min \left\{\delta_{k}, \delta_{k^{\prime}}\right\}$ units of flow on the new edges of $P_{j^{\prime}}\left(k, k^{\prime}\right)$. The new flow remains nested because an already existing routing path with positive flow is selected. The cost does not increase because the total edge cost of $P_{j^{\prime}}\left(k, k^{\prime}\right)$ is upper bounded by that of any $P_{j}\left(k, k^{\prime}\right), j \in J$. 
Finding a matching pair takes $O(n)$ time. An execution of the preceding procedure requires $O(\sigma)$ time. Every time the procedure is invoked for a matching pair $u_{k}$ and $u_{k^{\prime}}$, the flow leaving $u_{k}$ or entering $u_{k^{\prime}}$ on original edges drops to 0 . Thus, the total time to pack flow using the preceding procedure takes $O\left(n^{2} \sigma\right)$ time. Consider the point in time when there exists no matching pair anymore. At every vertex $u_{k^{\prime}}$ where the flow increases by injections from original edges, the flow is routed on path $P_{\sigma}\left(1, k^{\prime}\right)$. At every vertex $u_{k}$ where the flow decreases by shipment along original edges, the flow is routed on path $P_{\sigma}(k, n)$. The flow on any of these paths is integral because at $u_{k^{\prime}}$ and $u_{k}$, the increase/decrease is integral. Thus, the remaining flow on original edges can be transferred without modification to the auxiliary edges. In summary, Step 3 takes $O\left(n^{2} \sigma\right)$ time.

Claim 3. For every $j \in J$, path $P_{j}\left(k, k^{\prime}\right)$ routes $\delta_{j}$ units of flow.

Proof. First, assume that $\delta_{k} \leq \delta_{k^{\prime}}$. Suppose that the statement of the claim does not hold. We derive a contradiction. In the following, we always analyze flow on original edges. By the definition of a matching pair, the flow on $\left(u_{k}, l_{j, k}\right)$, which then traverses lower path $j$, is not shipped to any vertex between $u_{k}$ and $u_{k^{\prime}}$ on the upper path. Hence, it reaches $l_{j, k^{\prime}}$. Let $j_{1} \in J$ be the smallest integer such that less than $f\left(u_{k}, l_{j_{1}, k}\right)$ units of flow are sent along $f\left(l_{j_{1}, k^{\prime}}, u_{k^{\prime}}\right)$. Hence, some flow entering $l_{j_{1}, k^{\prime}}$ must continue on lower path $j_{1}$. This implies the existence of a path $P_{j_{1}}\left(k, k_{1}\right)$, with $k^{\prime}<k_{1}$, that routes flow. Considering that a total of at least $\delta_{k}$ units of flow are shipped to $u_{k^{\prime}}$, some flow must be routed from a vertex $u_{k_{2}}$ with $k_{2}<k$, or from $l_{\sigma, 1}$ at the beginning of the component. Hence, there must exist a path $P_{j}\left(k_{2}, k^{\prime}\right), j \neq j_{1}$, that routes flow. We obtain a contradiction to the fact that the flow is nested because $P_{j_{1}}\left(k, k_{1}\right)$ ships flow and $k_{2}<k<k^{\prime}<k_{1}$. The case $\delta_{k}>\delta_{k^{\prime}}$ is analogous.

\subsection{Construction of the Schedule}

Let $f^{3}$ denote the flow obtained in Step 3. We need two lemmas. The following Lemma 4.6 relates $f^{3}$ to schedules with $\tau m_{i}$ servers of type $i, 1 \leq i \leq \tau$.

Lemma 4.6. Flow $f^{3}$ corresponds to a schedule $\Sigma$ with $\tau m_{i}$ servers of type $i, 1 \leq i \leq \tau$. In $\left[t_{k}, t_{k+1}\right)$, exactly $d_{i, k}=\min \left\{m_{i},\left\lfloor\tau f^{*}\left(u_{i, k}, u_{i, k+1}\right)\right\rfloor\right\}$ servers of type $i$ are in the active state, $1 \leq i \leq \tau$ and $1 \leq$ $k \leq n-1$. The energy consumed by the servers of type $i$ is equal the cost of $f^{3}$ in $C_{i}, 1 \leq i \leq \tau$.

Proof. For any component $C_{i}$ of $\mathcal{N}(\mathcal{I}), 1 \leq i \leq \tau$, consider the flow $f^{3}$. It ships $\tau m_{i}$ flow units through $C_{i}$. Along edge $\left(u_{i, k}, u_{i, k+1}\right)$, exactly $d_{i, k}$ units of flow are routed. Considering that the flow is integral, it can be decomposed into $\tau m_{i}$ unit flows/paths through $C_{i}$ : Start at vertex $l_{i, \sigma_{i}, 1}$. At any vertex $v$, traverse an arbitrary outgoing edge $e=(v, w)$ with positive flow and remove one unit from it. A path ends at $l_{i, \sigma_{i}, n}$. Each of these paths corresponds to a schedule for a server of type $i$. The energy consumed by the server is equal to the cost incurred by the flow unit/path. Considering that $d_{i, k}$ units of flow are sent along $\left(u_{i, k}, u_{i, k+1}\right), d_{i, k}$ servers are in the active state during $\left[t_{k}, t_{k+1}\right), 1 \leq k \leq n-1$.

The next Lemma 4.7 allows us to reduce a schedule with $\tau m_{i}$ servers of type $i$, where $1 \leq i \leq \tau$, to one with only $m_{i}$ servers of this type. At any time, the lowest numbered servers are in the active state.

Lemma 4.7. Let $\Sigma_{i}$ be a schedule for $\tau m_{i}$ servers of type $i$ in which exactly $d_{i, k}$ servers are in the active state during $\left[t_{k}, t_{k+1}\right)$, where $d_{i, k} \leq m_{i}$ and $1 \leq k \leq n-1$. Then there exists a schedule $\Sigma_{i}^{\prime}$ for $m_{i}$ servers of type $i$ in which the servers numbered 1 to $d_{i, k}$ are in the active state during $\left[t_{k}, t_{k+1}\right)$. The energy consumed by $\Sigma_{i}^{\prime}$ is upper bounded by that of $\Sigma_{i}$.

Proof. Given $\Sigma_{i}$, we modify it such that, among the $\tau m_{i}$ servers, those numbered 1 to $d_{i, k}$ are active in $\left[t_{k}, t_{k+1}\right)$, for $k=1, \ldots, n-1$. The modifications never increase the consumed energy. Let 
$\left[t_{k}, t_{k+1}\right)$ be the first interval such that at least one server is active. Determine the set $I$ of server numbers such that the respective servers reside in the active state during $\left[t_{k}, t_{k+1}\right)$. Construct an arbitrary one-to-one mapping $\pi$ between $\left\{1, \ldots, d_{i, k}\right\}$ and $I$. In $\Sigma_{i}$, replace the server numbered $\pi(h)$ by that numbered $h$ and vice versa, for any $h \in I$. More specifically, in the entire schedule, server $h$ assumes the states and performs the state transitions of server $\pi(h)$ and vice versa, for any $h \in I$. These swaps are just renaming operations that do not affect the energy consumed by the schedule.

Let $\Sigma_{i}$ be a schedule for $\tau m_{i}$ servers such that during any interval $\left[t_{k^{\prime}}, t_{k^{\prime}+1}\right)$, the servers numbered 1 to $d_{i, k^{\prime}}$ are in the active state, for $k^{\prime}=1, \ldots, k-1$. We assume that in low-power mode, a server never changes state. Otherwise, we can change schedule $\Sigma_{i}$ accordingly, without increasing its cost; cf. Proposition 2.1. We consider interval $\left[t_{k}, t_{k+1}\right)$. If the servers residing in the active state are not those numbered 1 to $d_{i, k}$, then let $h \geq 1$ be the smallest integer such that server $h$ is not active. Let $h^{\prime}$ be the highest server number such that the respective server is active. Let $s_{i, j}$ be the low-power state of server $h$ at time $t_{k}$. We distinguish two cases.

Suppose that server $h$ was active during $\left[t_{k-1}, t_{k}\right)$. If server $h^{\prime}$ was also active during $\left[t_{k-1}, t_{k}\right)$, then we change the schedule in the following way. At time $t_{k}$, server $h$ remains active and $h^{\prime}$ transitions to low-power state $s_{i, j}$. If server $h^{\prime}$ was not active during $\left[t_{k-1}, t_{k}\right)$, then let $s_{i, j^{\prime}}$ be the state of the server during that interval. If $j^{\prime} \geq j$, server $h^{\prime}$ simply remains in state $s_{i, j^{\prime}}$ at time $t_{k}$. Otherwise, let $t^{\prime}$ be the most recent time when server $h^{\prime}$ transitioned to $s_{i, j^{\prime}}$. We modify the schedule such that server $h^{\prime}$ transitions to state $s_{i, j}$ at time $t^{\prime}$. Starting at time $t_{k}$, we interchange the roles of servers $h$ and $h^{\prime}$ in the rest of the schedule.

Next, assume that server $h$ was not active during $\left[t_{k-1}, t_{k}\right)$. Then by induction hypothesis, server $h^{\prime}$ was not active either. Let $s_{i, j^{\prime}}$ be the state of the server $h^{\prime}$ during $\left[t_{k-1}, t_{k}\right)$. We change the schedule as follows. At time $t_{k}$, server $h$ powers up while server $h^{\prime}$ remains in low-power mode. If $j>j^{\prime}$, we additionally have to change low-power states. Again, let $t^{\prime}$ be the most recent time when server $h^{\prime}$ transitioned to $s_{i, j^{\prime}}$. Similarly, let $t$ be the most recent time when server $h$ powered down. By induction hypothesis, $t^{\prime} \leq t$. If $j>j^{\prime}$, then at time $t^{\prime}$ server $h^{\prime}$ powers down to $s_{i, j}$. At time $t$, server $h$ transitions to $s_{i, j^{\prime}}$.

In the schedule obtained after the preceding transformation, the highest numbered $(\tau-1) m_{i}$ servers are never active. Hence, we can simply remove them; the consumed energy can only decrease.

Given the integral flow $f^{3}$, algorithm $\mathcal{A}_{2}$ constructs a feasible schedule $\Sigma^{*}$ for $\mathcal{I}$. For each server type $\mathcal{S}_{i}, 1 \leq i \leq \tau, \mathcal{A}_{2}$ builds an optimal schedule $\Sigma_{i}^{*}$ such that $d_{i, k}$ of the $m_{i}$ servers in $\mathcal{S}_{i}$ are in the active state during $\left[t_{k}, t_{k+1}\right), 1 \leq k \leq n-1$. These schedules $\Sigma_{1}^{*}, \ldots, \Sigma_{\tau}^{*}$ are then combined to form $\Sigma^{*}$. More specifically, consider any $i$ with $1 \leq i \leq \tau$. In a first step, given Lemma $4.7, \Sigma_{i}^{*}$ just specifies that the servers numbered 1 to $d_{i, k}$ are in the active state during $\left[t_{k}, t_{k+1}\right)$, for any $1 \leq k \leq n-1$. Then, while a server is not required to be active according to the specification, $\mathcal{A}_{2}$ selects an optimal state. Suppose that at time $t_{k}$ the number of required servers decreases, i.e., $d_{i, k-1}>d_{i, k}$. Algorithm $\mathcal{A}_{2}$ determines states for $d_{i, k-1}-d_{i, k}$ servers that may power down. This is done as follows. Initially, $\mu:=d_{i, k}$. While $\mu<d_{i, k-1}, \mathcal{A}_{2}$ finds the next time $t_{k^{\prime}}$ such that $d_{i, k^{\prime}} \geq \mu+1$, i.e., at least $\mu+1$ servers are active. It chooses an optimal state to be assumed by servers numbered $\mu+1, \ldots, \min \left\{d_{i, k-1}, d_{i, k^{\prime}}\right\}$ at time $t_{k}$. This is the state $s_{i, j^{*}}$ with $j^{*}=\arg \min _{1 \leq j \leq \sigma_{i}}\left\{r_{i, j}\left(t_{k^{\prime}}-t_{k}\right)+\right.$ $\left.\Delta_{i, j}\right\}$. Then $\mu:=\min \left\{d_{i, k-1}, d_{i, k^{\prime}}\right\}$.

By Lemmas 4.6 and 4.7 , the energy consumed by $\Sigma_{i}^{*}$ is upper bounded by the cost incurred by $f^{3}$ in component $C_{i}$. Thus, the energy consumed by the combined schedule $\Sigma^{*}$ is at most $\operatorname{cost}\left(f^{3}\right) \leq$ $\tau \cos t\left(f^{*}\right)$. Schedule $\Sigma^{*}$ is feasible because, by Lemma $4.4, \sum_{i=1}^{\tau} d_{i, k} \geq d_{k}$. The proof of Theorem 4.1 is complete. 


\section{CONCLUSION}

In this article, we have introduced the problem DPM that models an important energy and capacity management problem in data centers. For the scenario that each server has exactly two states-an active state and a sleep state-we have developed a combinatorial, polynomial time algorithm for computing optimal schedules. For the setting where each server has an arbitrary number of states, we have devised a $\tau$-approximation algorithm, where $\tau$ is the number of server types.

There are several interesting directions for future research. A challenging problem is to settle the complexity of DPM if each server has an arbitrary number of states. We conjecture that the problem is NP-hard but have not been successful in developing a proof. Another working direction is to improve the approximation factor of $\tau$, assuming that the problem is indeed NP-hard. Otherwise, a polynomial time algorithm for computing optimal schedules has to be developed. Furthermore, it is sensible to study the online scenario where the computing demands are revealed over time.

In our problem DPM, the servers have equal computation power. It is interesting to explore a refined setting in which the servers have different computation power, which is related to their energy consumption. Specifically, if a server has a high computation power, then its energy consumption rate in the active state is high. Last but not least, in Section 1, we mentioned multicore architectures as a possible application of DPM. In these environments, the processors often have several active states. It would be interesting to model and algorithmically investigate such settings as well.

\section{APPENDIX}

Proof of Proposition 2.1. Consider any schedule. Let $\left[t, t^{\prime}\right)$ be a time interval such that $S_{i}$ powers down at time $t$ and is in low-power mode until time $t^{\prime}$. Let $j, 1 \leq j \leq \sigma_{i}$, be the largest integer such that $S_{i}$ visits $s_{i, j}$ between $t$ and $t^{\prime}$. Considering that the state transition energies satisfy the triangle inequality, the total energy needed to transition from the active state to $s_{i, j}$ is at least $\Delta_{i, 0, j}$. The total energy consumed in transitioning from $s_{i, j}$ to the active state is at least $\Delta_{i, j, 0}$. The server's states are numbered in order of decreasing power consumption rates. Hence, between $t$ and $t^{\prime}, S_{i}$ consumes the least energy if it always resides in $s_{i, j}$ and transitions between the active state and $s_{i, j}$ at times $t$ and $t^{\prime}$, respectively.

Proof of Proposition 2.2. Let $\Sigma$ be any optimal schedule that fulfills the property of Proposition 2.1. Suppose that it performs state transitions at times not contained in $T$. We show how to repeatedly modify $\Sigma$ so that it satisfies the properties of Proposition 2.2. Let $t \in\left[t_{1}, t_{n}\right)$ be the earliest time such that $t \notin T$ and a server $S_{i}$ performs a state transition at this time. Assume that $t \in\left(t_{k}, t_{k+1}\right)$, for some $1 \leq k \leq n-1$.

In a first step, we argue that if $S_{i}$ powers up at time $t$, then there must be another server that powers down at time $t$. Analogously, if $S_{i}$ powers down at time $t$, then there must be a server that powers up at time $t$. First, assume that $S_{i}$ powers up and that there is no other server that powers down at time $t$. Let $t^{\prime}$ be the earliest time such that $t^{\prime}>t$ and a state transition is performed at time $t^{\prime}$. Schedule $\Sigma$ meets the computing demand during $\left[t_{k}, t\right)$, which do not change until $t_{k+1}$. Furthermore, no server powers down during $\left(t, t^{\prime}\right)$. Hence, at time $t$, server $S_{i}$ can remain in its current low-power state until time $\min \left\{t_{k+1}, t^{\prime}\right\}$ and then power up. The modified schedule is feasible and has a strictly smaller energy consumption than $\Sigma$. This contradicts the fact that $\Sigma$ is optimal. Next, suppose that $S_{i}$ powers down and that no other server powers up at time $t$. Schedule $\Sigma$ meets the computing demand at time $t$, which is identical to that in $\left[t_{k}, t\right)$. By the choice of $t$, no state transitions occur during $\left(t_{k}, t\right)$. Thus, $S_{i}$ can power down already at time $t_{k}$. The resulting schedule is feasible and consumes less energy than $\Sigma$. 
Therefore, suppose that $S_{i}$ powers up and that some server $S_{i^{\prime}}$ powers down at time $t$. Let $s_{i, j}$, with $1 \leq j \leq \sigma_{i}$, be the state in which $S_{i}$ resides just before powering up at time $t$. Let $s_{i^{\prime}, j^{\prime}}, 1 \leq j^{\prime} \leq$ $\sigma_{i^{\prime}}$, be the state to which $S_{i^{\prime}}$ transitions at time $t$. A first observation is that $r_{i, 0}-r_{i, j}=r_{i^{\prime}, 0}-r_{i^{\prime}, j^{\prime}}$. If $r_{i, 0}-r_{i, j}<r_{i^{\prime}, 0}-r_{i^{\prime}, j^{\prime}}$ held true, then $S_{i}$ and $S_{i^{\prime}}$ could perform their state transitions already at time $t-\delta$, where $\delta>0$ is chosen such that $t_{k} \leq t-\delta$. The energy consumed by the new schedule is equal to that of the original one plus $\delta\left(r_{i, 0}-r_{i, j}+r_{i^{\prime}, j^{\prime}}-r_{i^{\prime}, 0}\right)$. The last expression is smaller than 0 . If $r_{i, 0}-r_{i, j}>r_{i^{\prime}, 0}-r_{i^{\prime}, j^{\prime}}$ held true, then $S_{i}$ and $S_{i^{\prime}}$ could postpone their state transitions to time $t+\delta$, where $\delta>0$ is chosen such that neither $S_{i}$ nor $S_{i^{\prime}}$ performs a state transition during $(t, t+\delta)$. The energy consumption of the new schedule is equal to that of the original one plus $\delta\left(r_{i^{\prime}, 0}-r_{i^{\prime}, j^{\prime}}+r_{i, j}-r_{i, 0}\right)$. Again, the last expression is smaller than 0 .

Let $t(i)$ be the earliest time such that $t(i)>t$ and $S_{i}$ powers down. Similarly, let $t\left(i^{\prime}\right)>t$ be the earliest time such that $S_{i^{\prime}}$ powers up. If $S_{i^{\prime}}$ does not power up again, let $t\left(i^{\prime}\right)=t_{n}$. We modify $\Sigma$. First, assume that $t(i)>t\left(i^{\prime}\right)$. In this case, at time $t$, server $S_{i}$ remains in $s_{i, j}$ until $t\left(i^{\prime}\right)$ and then powers up. Server $S_{i^{\prime}}$ remains in the active state until $t\left(i^{\prime}\right)$. Considering that $r_{i, 0}-r_{i, j}=r_{i^{\prime}, 0}-r_{i^{\prime}, j^{\prime}}$ and $S_{i^{\prime}}$ does not perform state transitions at times $t$ and $t\left(i^{\prime}\right)$, the energy consumption of the modified schedule cannot be larger than that of the original one.

Next, assume that $t(i) \leq t\left(i^{\prime}\right)$. The schedule modifications are as follows. Server $S_{i^{\prime}}$ remains in the active state until time $t(i)$. It then powers down to $s_{i^{\prime}, j^{\prime}}$ if $t(i)<t\left(i^{\prime}\right)$ or if $t(i)=t\left(i^{\prime}\right)=t_{n}$. Let $s_{i, j^{\prime \prime}}$, with $1 \leq j^{\prime \prime} \leq \sigma_{i}$, be the state selected by $S_{i}$ at time $t(i)$. If $j \geq j^{\prime \prime}$, then $S_{i}$ remains in state $s_{i, j}$ at time $t$ until the next time $S_{i}$ powers up, if such a time exists. If $j<j^{\prime \prime}$, then at the most recent time at which $S_{i}$ transitioned to $s_{i, j}$, it selects $s_{i, j^{\prime \prime}}$ instead. It remains in this state until the next powering up. The total energy consumed by $S_{i}$ and $S_{i^{\prime}}$ while residing in the various states does not increase. For the interval from $t$ to $t(i)$, this holds true because, again, $r_{i, 0}-r_{i, j}=r_{i^{\prime}, 0}-r_{i^{\prime}, j^{\prime}}$ and $r_{i, j^{\prime \prime}}<r_{i, j}$ if $j<j^{\prime \prime}$. In fact, if $j<j^{\prime \prime}$, then an energy of $r_{i, j}-r_{i, j^{\prime \prime}}$ is saved during time units prior to $t(i)$. If $j>j^{\prime \prime}$, then $r_{i, j}<r_{i, j^{\prime \prime}}$ and an energy of $r_{i, j^{\prime \prime}}-r_{i, j}$ is saved during time units after $t(i)$. The energy incurred by state transitions does not increase either. For $S_{i^{\prime}}$, this is obvious. If $S_{i^{\prime}}$ originally performed a power-up operation at time $t(i)$, then the corresponding energy is saved in the modified schedule. Server $S_{i}$ does not perform state transitions at times $t$ and $t(i)$. Hence, if $j \geq j^{\prime \prime}$, then an energy of $\Delta_{i, j^{\prime \prime}, 0}$ is saved. If $j<j^{\prime \prime}$, then an energy of $\Delta_{i, j, 0}$ is saved.

By repeating the modifications described in the last paragraph, we obtain a schedule in which no state transitions occur at time $t$. State transitions performed at times $t^{\prime}<t$ still satisfy $t^{\prime} \in T$. Handling further times $t \notin T$ with state transitions in the same way, we obtain a final schedule with the desired properties.

\section{REFERENCES}

[1] Ravindra K. Ahuja, Thomas L. Magnanti, and James B. Orlin. 1993. Network Flows: Theory, Algorithms, and Applications. Prentice Hall.

[2] Michael Armbrust, Armando Fox, Rean Griffith, Anthony D. Joseph, Randy H. Katz, Andrew Konwinski, Gunho Lee, et al. 2009. Above the Clouds: A Berkeley View of Cloud Computing. Technical Report UCB/EECS-2009-28. EECS Department, University of California, Berkeley.

[3] John Augustine, Sandy Irani, and Chaitanya Swamy. 2008. Optimal power-down strategies. SIAM fournal on Computing 37, 5 (2008), 1499-1516.

[4] Yossi Azar, Yair Bartal, Esteban Feuerstein, Amos Fiat, Stefano Leonardi, and Adi Rosén. 1999. On capital investment. Algorithmica 25, 1 (1999), 22-36.

[5] Luiz André Barroso and Urs Hölzle. 2007. The case for energy-proportional computing. IEEE Computer 40, 12 (2007), 33-37.

[6] Wikipedia. 2019. Heterogeneous Computing. Retrieved October 1, 2019 from https://en.wikipedia.org/wiki/ Heterogeneous_computing.

[7] United Extensible Firmware Interface Forum. 2016. The Advanced Configuration and Power Interface. The latest specification 6.1 is available at https://UEFI.org. 
[8] Miyuru Dayarathna, Yonggang Wen, and Rui Fan. 2016. Data center energy consumption modeling: A survey. IEEE Communications Surveys and Tutorials 18, 1 (2016), 732-794.

[9] Erik D. Demaine, Mohammad Ghodsi, Mohammad Taghi Hajiaghayi, Amin S. Sayedi-Roshkhar, and Morteza Zadimoghaddam. 2013. Scheduling to minimize gaps and power consumption. fournal of Scheduling 16, 2 (2013), 151-160.

[10] Gerhard Fettweis and Ernesto Zimmermann. 2008. ICT energy consumption-Trends and challenges. In Proceedings of the 11th International Symposium on Wireless Personal Multimedia Communications. 1-6.

[11] Anshul Gandhi and Mor Harchol-Balter. 2011. How data center size impacts the effectiveness of dynamic power management. In Proceedings of the Annual Allerton Conference on Communication, Control, and Computing. 11641169.

[12] Anshul Gandhi, Mor Harchol-Balter, and Ivo J. B. F. Adan. 2010. Server farms with setup costs. Performance Evaluation 67, 11 (2010), 1123-1138.

[13] Zygmunt J. Haas and Shuyang Gu. 2015. On power management policies for data centers. In Proceedings of the IEEE International Conference on Data Science and Data Intensive Systems (DSDIS'15). 404-411.

[14] Ward Van Heddeghem, Sofie Lambert, Bart Lannoo, Didier Colle, Mario Pickavet, and Piet Demeester. 2014. Trends in worldwide ICT electricity consumption from 2007 to 2012. Computer Communications 50 (2014), 64-76.

[15] Sandy Irani, Sandeep K. Shukla, and Rajesh K. Gupta. 2003. Online strategies for dynamic power management in systems with multiple power-saving states. ACM Transactions on Embedded Computing Systems 2, 3 (2003), 325-346.

[16] Anna R. Karlin, Claire Kenyon, and Dana Randall. 2003. Dynamic TCP acknowledgment and other stories about e/(e-1). Algorithmica 36, 3 (2003), 209-224.

[17] Anna R. Karlin, Mark S. Manasse, Larry Rudolph, and Daniel Dominic Sleator. 1988. Competitive snoopy caching. Algorithmica 3 (1988), 77-119.

[18] Samir Khuller, Jian Li, and Barna Saha. 2010. Energy efficient scheduling via partial shutdown. In Proceedings of the 21st Annual ACM-SIAM Symposium on Discrete Algorithms (SODA'10). 1360-1372.

[19] Amir Levi and Boaz Patt-Shamir. 2015. Non-additive two-option ski rental. Theoretical Computer Science 584 (2015), $42-52$.

[20] Jian Li and Samir Khuller. 2011. Generalized machine activation problems. In Proceedings of the 22nd Annual ACMSIAM Symposium on Discrete Algorithms (SODA'11). 80-94.

[21] Minghong Lin, Adam Wierman, Lachlan L. H. Andrew, and Eno Thereska. 2013. Dynamic right-sizing for powerproportional data centers. IEEE/ACM Transactions on Networking 21, 5 (2013), 1378-1391.

[22] Zvi Lotker, Boaz Patt-Shamir, and Dror Rawitz. 2012. Rent, lease, or buy: Randomized algorithms for multislope ski rental. SIAM Journal on Discrete Mathematics 26, 2 (2012), 718-736.

[23] Kai Wang, Minghong Lin, Florin Ciucu, Adam Wierman, and Chuang Lin. 2015. Characterizing the impact of the workload on the value of dynamic resizing in data centers. Performance Evaluation 85-86 (2015), 1-18.

Received September 2017; revised March 2019; accepted April 2019 